\title{
A Polyhedral Bound on the Indeterminate Contact Forces in Planar Quasi-Rigid Fixturing and Grasping Arrangements
}

\author{
Elon Rimon, Member, IEEE, Joel W. Burdick, and Toru Omata, Member, IEEE
}

\begin{abstract}
This paper considers multiple-contact arrangements where several bodies grasp, fixture, or support an object via frictional point contacts. Within a strictly rigid-body modeling paradigm, when an external wrench (i.e., force and torque) acts on the object, the reaction forces at the contacts are typically indeterminate and span an unbounded linear space. This paper analyzes the contact reaction forces within a generalized quasi-rigid-body framework that keeps the desirable geometric properties of rigidbody modeling, while also including more realistic physical effects. We describe two basic principles that govern the contact mechanics of quasi-rigid bodies. The main result is that for any given external wrench acting on a quasi-rigid object, the statically feasible contact reaction forces lie in a bounded polyhedral set that depends on the external wrench, the grasp's geometry, and the preload forces. Moreover, the bound does not depend upon any detailed knowledge of the contact mechanics parameters. When some knowledge of the parameters is available, the bound can be sharpened. The polyhedral bound is useful for "robust" grasp and fixture synthesis. Given a set of external wrenches that may act upon an object, the grasp's geometry and preload forces can be chosen such that all of these external wrenches would be automatically supported by the contacts.
\end{abstract}

Index Terms-Fixturing, grasping, polyhedral bound, statically indeterminate contact forces.

\section{INTRODUCTION}

$\mathbf{T}$ HIS paper considers multiple-contact arrangements where an object is grasped, fixtured, or supported in static equilibrium by several bodies via frictional point contacts. Under an ideal rigid-body assumption, the reaction forces at the contacts due to an external wrench acting on the object are typically indeterminate and span an unbounded linear space. For instance, in generic $k$-contact planar grasps, the indeterminate contact forces span a $(2 k-3)$-dimensional linear space. This indeterminacy is an artifact of the simplicity of rigid-body models, and it can cause difficulties in the analysis, synthesis, and implementation of reliable grasping and fixturing systems. However,

Manuscript received July 23, 2003; revised June 30, 2004. This paper was recommended for publication by Associate Editor Y. H. Liu and Editor I. Walker upon evaluation of the reviewers' comments. This work was supported by the National Science Foundation under Grant 9901056. This paper was presented in part at the IEEE International Conference on Robotics and Automation, Taiwan, R.O.C., 2003.

E. Rimon is with the Department of Mechanical Engineering, Technion-Israel Institute of Technology, Haifa 32000, Israel (e-mail: elon@ robby.technion.ac.il).

J. W. Burdick is with the Department of Mechanical Engineering, California Institute of Technology, Pasadena, CA 91125 USA (e-mail: jwb@ robotics.caltech.edu).

T. Omata is with the Department of Mechanical Engineering, Tokyo Institute of Technology, Tokyo 152-9550, Japan (e-mail: omata@ pms.titech.ac.jp).

Digital Object Identifier 10.1109/TRO.2005.862478 in reality, all bodies possess some degree of natural compliance due to material deformation at the contacts. ${ }^{1}$ These elastic deformations induce specific contact forces in response to an applied external wrench. Unfortunately, the laws governing compliant deformation depend on various geometric and material properties of the contacting bodies [15], [35]. If specific values of these parameters are known, then the reaction forces can often be uniquely determined. However, a detailed knowledge of these properties is often not readily available in practice, and measurement of these properties requires sophisticated sensing capabilities. Clearly, one would like a theory to predict contact reaction forces that retains the attractive geometric properties of rigid-body models, while also offering predictions that better match reality. Moreover, the theory should be "robust" in that it does not depend heavily upon detailed knowledge of the contact parameters or specific assumptions about the underlying compliant model.

This paper takes a middle-ground approach between rigidbody idealization and conventional compliant contact models. We introduce two generic rules governing the mechanics of quasi-rigid-body contact: a micro-penetration principle that determines the change in the normal component of the contact forces and a micro-slip principle that determines the change in the tangential or frictional component of the contact forces. Put simply, these two principles are generic normal and tangential compliance laws whose basic properties hold for all quasi-rigidbody compliance models. We stress that detailed knowledge of contact parameters (such as material stiffness) or compliance models are not needed to apply these principles. Hence, our approach can be seen as a form of robust contact modeling. Coupling the effects of these principles at the individual contacts through the rigid-body kinematics of the grasped object yields a polyhedral bound on the indeterminate reaction forces. Moreover, the polyhedral bound does not depend on specific knowledge of the physical contact parameters, thus allowing grasp analysis and synthesis under huge uncertainty in these parameters. Because we allow for large parametric uncertainty, the indeterminacy in the contact forces cannot be eliminated, but can instead be bounded by a polyhedral set, whose connectivity, convexity, and number of bounding vertices are analyzed.

Relationship to Prior Work: The indeterminate forces arising from rigid-body analysis are in part an artifact of the rigid-body modeling paradigm. To measure the actual reac-

\footnotetext{
${ }^{1}$ Significant compliance may also exist in the joints of an articulated grasping mechanism. While these effects are not considered here, they could be analyzed by extensions of the method proposed in this paper.
} 
tion forces that arise in an application, thereby resolving the indeterminate forces predicted by offline analysis, one can install force sensors at the contacts, e.g., [6], [11], [17], [18], and [40]. While this approach is useful for controlling the contact forces, it does not provide any analytical insight or prediction as to what actual forces might appear in a given application. The analytical study of the indeterminate contact forces has been motivated by power grasp and whole-arm manipulation applications [2], [24], [36], [43]. ${ }^{2}$ In the context of these applications, two approaches were proposed to deal with the indeterminacy of rigid-body models. The first approach implements compliant behavior using some suitable stiffness-control method [7], [11], [27], [32]. The stiffness matrix of the resulting grasping mechanism can then predict the reaction forces due to external loads acting on the object [3]. A second approach is to measure the joint torques of the grasping mechanism as a means for resolving the indeterminate forces [2], [29], [31]. Unfortunately, Bicchi [2] has shown that the kinematics of the grasping mechanism induces a subspace of indeterminate forces that cannot be measured by joint torques. In addition to static indeterminacy, the rigid-body modeling paradigm is sometimes ambiguous in predicting the dynamic response of a grasped object to an applied wrench [9], [19]. Trinkle calls a contact arrangement strongly stable with respect to an applied wrench if the only dynamically feasible response is the one where all contacts remain stationary [30]. While strong stability deals with dynamic ambiguity that can arise in rigid-body models, it does not address the static indeterminacy of the contact forces which is the subject of this paper.

The polyhedral bound reported here builds upon earlier work by the third author in the area of power grasps [28], [29]. Under a reasonable assumption of one contact per link, Omata reported that the tangential component of the indeterminate contact forces lie in a bounded polyhedral set. Our analysis differs from this earlier work in three fundamental ways. First, we ignore the grasping mechanism and focus solely on the interaction between the grasped object and its surrounding bodies. The polyhedral bound reported here is consequently useful in applications where joint torques are not necessarily available, such as fixturing and industrial gripping applications. Second, the third author previously assumed that the contacting bodies are perfectly rigid. In contrast, we assume that the bodies are quasi-rigid and can locally deform at the contacts. Third, Omata previously focused on bounding the tangential component of the indeterminate contact forces. We provide a bound for both the tangent and normal component of the contact forces.

An important potential application of the polyhedral bound is the following grasp synthesis approach. We are given a bounded collection of external wrenches that can act on the object, as well as a conservative lower bound on the coefficient of friction at the contacts. Using these two specifications, we can select the grasp's geometry and preload forces such that the entire bounded set of indeterminate reaction forces induced by the external wrenches satisfies the friction-cone constraints at the

\footnotetext{
${ }^{2}$ In these applications, an object is manipulated by one or more articulated mechanisms which are allowed to establish multiple midlink contacts with the manipulated object [4].
}

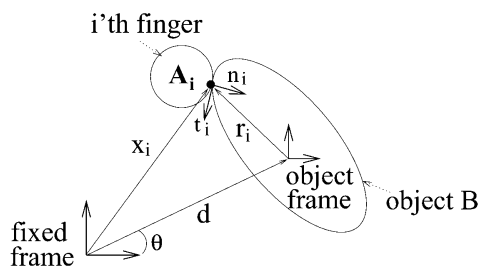

Fig. 1. $\left(d_{x}, d_{y}, \theta\right)$ parametrization of $\mathcal{B}$ 's c-space.

contacts, even in the presence of huge contact parameter uncertainty. Moreover, the resulting grasp will automatically cancel all external wrenches in the given collection, without any of the contacts slipping or breaking away from the object. This cancellation occurs strictly due to mechanical effects and does not require active control of the contact forces. Note that existing grasp synthesis approaches establish preloaded grasps that resist only some unspecified local neighborhood of external wrenches centered at the origin [14], [21], [23], [25], [39]. In contrast, our synthesis approach generates grasps which are guaranteed to resist an entire specified collection of external wrenches without resorting to active contact force control.

The paper is organized as follows. In Section II, we describe the principles of micro-penetration and micro-slip governing the mechanics of quasi-rigid contacts. In Section III, we derive the polyhedral bound on the contact forces by coupling the contact-wise effect of the two principles through the rigid body kinematics of the grasped object. Section IV demonstrates the polyhedral bound on concrete examples, highlighting various properties of the bound. To motivate the utility of the polyhedral bound, Section V sketches a grasp synthesis approach which is based on the polyhedral bound, demonstrating the approach with an example. The concluding section discusses an extension of the polyhedral bound to three dimensions. Finally, the appendices contain proof details, as well as a polynomial time algorithm for computing the polyhedral bound.

\section{Micro-Penetration AND Micro-SLiP PrinCIPLES}

This section introduces basic grasping terminology and then describes two principles that govern the possible changes in the normal and tangential component of the contact forces when an external wrench is applied to a quasi-rigid body.

\section{A. Grasping Terminology}

We assume that a planar object $\mathcal{B}$ is in frictional point contact with stationary planar bodies $\mathcal{A}_{1}, \ldots, \mathcal{A}_{k}$. The bodies $\mathcal{A}_{1}, \ldots, \mathcal{A}_{k}$ represent fingertips or fixturing elements, but we shall simply call them "fingers." The configuration space (c-space) of a planar object $\mathcal{B}$ is parametrized by $q=\left(d_{x}, d_{y}, \theta\right) \in \mathbb{R}^{3}$, where $d=\left(d_{x}, d_{y}\right)$ parametrizes translation and $\theta$ parametrizes the orientation of $\mathcal{B}$ (Fig. 1). The linear and angular velocity of $\mathcal{B}$ at a configuration $q$ is represented by a tangent vector $\dot{q} \in T_{q} \mathbb{R}^{3}$, where $T_{q} \mathbb{R}^{3}$ is the tangent space of $\mathbb{R}^{3}$ at $q$. Let $x_{i}$ denote the contact point between $\mathcal{A}_{i}$ and $\mathcal{B}$, which is expressed in a fixed reference frame. Let $r_{i}$ denote the same point when expressed in $\mathcal{B}$ 's body frame (Fig. 1). Then, $x_{i}$ is related to $r_{i}$ by the rigid-body transformation: $x_{i}=X\left(r_{i}, q\right)=R(\theta) r_{i}+d$, where 
$R(\theta)$ is the orientation matrix of $\mathcal{B}$. Let $X_{r_{i}}(q)$ denote the rigid-body transformation with $r_{i}$ held fixed. When $\mathcal{B}$ moves along a c-space trajectory $q(t)$, the velocity of $X_{r_{i}}$ is given by $(d / d t) X_{r_{i}}(q(t))=G_{i}^{T} \dot{q}(t)$, where $G_{i}^{T}$ is the $2 \times 3$ matrix given by $G_{i}^{T}=\left[I J \rho_{i}\right]$, where $I$ is a $2 \times 2$ identity matrix

$$
J=\left[\begin{array}{cc}
0 & -1 \\
1 & 0
\end{array}\right]
$$

and $\boldsymbol{\rho}_{i}=R(\theta) r_{i}$.

Contact Force Space: Let $\boldsymbol{f}=\left(\boldsymbol{f}_{1}, \ldots, \boldsymbol{f}_{k}\right)$ denote the finger forces at the $k$ contacts, which are expressed with respect to a fixed world frame. The tangent and normal components of the contact forces are denoted $\left(\left(f_{1}^{t}, f_{1}^{n}\right), \ldots,\left(f_{k}^{t}, f_{k}^{n}\right)\right) \in \mathbb{R}^{2 k}$, and the space having these coordinates is called contact force space. The coordinate representation of $f$ is obtained as follows. Let $n_{i}$ denote the unit normal to the boundary of $\mathcal{A}_{i}$ and $\mathcal{B}$ at $x_{i}$, pointing into $\mathcal{B}$ (Fig. 1). Let $t_{i}$ denote the unit tangent to the boundary of $\mathcal{A}_{i}$ and $\mathcal{B}$ at $x_{i}$, such that $t_{i}$ and $n_{i}$ form a right-handed system. Then, $f_{i}^{t}=t_{i} \cdot \boldsymbol{f}_{i}$ and $f_{i}^{n}=n_{i} \cdot \boldsymbol{f}_{i}$. The following tangent and normal projection matrices project the contact forces onto their tangent and normal coordinates:

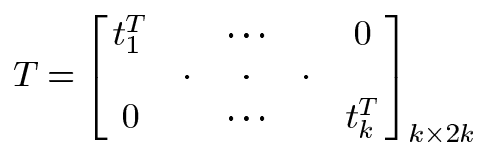

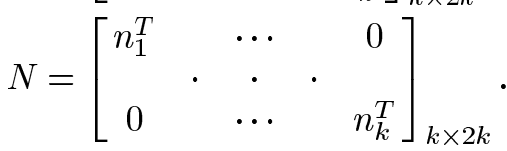

Thus, $f^{t}=T \boldsymbol{f}$ and $f^{n}=N \boldsymbol{f}$, where $f^{t}=\left(f_{1}^{t}, \ldots, f_{k}^{t}\right)$ and $f^{n}=\left(f_{1}^{n}, \ldots, f_{k}^{n}\right)$. Finally, the wrench induced on $\mathcal{B}$ by a force $\boldsymbol{f}_{i}$ acting at $x_{i}$, denoted $\boldsymbol{w}_{i}$, is given by

$$
\boldsymbol{w}_{i}=\left(\begin{array}{c}
\boldsymbol{f}_{i} \\
\boldsymbol{\rho}_{i}^{T} J^{T} \boldsymbol{f}_{i}
\end{array}\right)=G_{i} \boldsymbol{f}_{i}, \quad \text { where } G_{i}=\left[\begin{array}{c}
I \\
\boldsymbol{\rho}_{i}^{T} J^{T}
\end{array}\right] .
$$

When $k$ bodies apply forces $\boldsymbol{f}=\left(\boldsymbol{f}_{1}, \ldots, \boldsymbol{f}_{k}\right)$ on $\mathcal{B}$, the net wrench acting on $\mathcal{B}$ is given by $\boldsymbol{w}=\sum_{i=1}^{k} G_{i} \boldsymbol{f}_{i}=G \boldsymbol{f}$, where $G=\left[G_{1} \cdots G_{k}\right]_{3 \times 2 k}$ is the well-known grasp matrix.

\section{B. Mechanics of Micro-Penetration}

We now formulate a generic rule for the change in the normal component of a contact force due to material deformation at the contact. The usual assumption made in the solid mechanics literature is that the contacting bodies are quasi-rigid, meaning that their deformations due to compliance effects are localized to the vicinity of the contacts [15], [35]. This assumption allows us to describe the overall motion of $\mathcal{B}$ relative to the stationary bodies $\mathcal{A}_{1}, \ldots, \mathcal{A}_{k}$ using rigid-body kinematics. The quasi-rigidity assumption is generally valid for all bodies which are not made of exceptionally soft material and do not contain slender substructures. In practice, quasi-rigidity is quite a satisfactory model when the normal deformations are significantly smaller than the characteristic radius of curvature of the contacting bodies [41].

A convenient lumped-parameter model for the mechanics of compliant contact is based on overlap functions [33]. This quasirigid-body modeling scheme can be described as follows. Consider a single contact between $\mathcal{B}$ and $\mathcal{A}_{\boldsymbol{i}}$. In the absence of deformation, the two bodies contact at a single point. When pushed

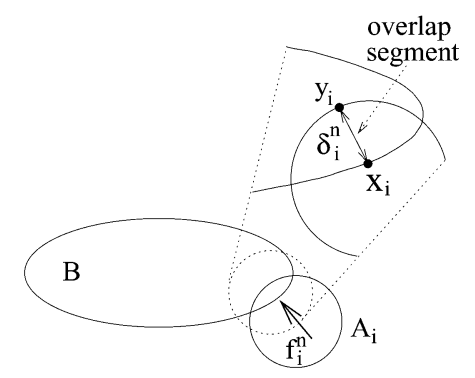

Fig. 2. Overlap segment representing the interpenetration of $\mathcal{A}_{i}$ and $\mathcal{B}$.

together, the two contacting surfaces deform. One can conceptually think of the two rigid bodies as interpenetrating or overlapping their undeformed shapes, as illustrated in Fig. 2. Let $\mathcal{B}$ be at a configuration $q$. Then, the overlap ${ }^{3}$ between $\mathcal{B}$ and $\mathcal{A}_{i}$, denoted $\delta_{i}^{n}(q)$, is the minimum amount of translation of $\mathcal{B}$ that would separate it from $\mathcal{A}_{i}$. By definition, $\delta_{i}^{n}(q)$ vanishes when $\mathcal{B}$ is disjoint from $\mathcal{A}_{i}$. The overlap segment is the segment whose endpoints lie on the boundary of $\mathcal{B}$ and $\mathcal{A}_{i}$, such that the length of the segment is $\delta_{i}^{n}$, and its orientation gives the direction of separating translation. It can be verified that for sufficiently small and positive $\delta_{i}^{n}$, the overlap segment is the unique segment collinear with the normals to the boundaries of $\mathcal{B}$ and $\mathcal{A}_{i}$ (Fig. 2). In this lumped-parameter form of modeling, the net normal force induced by the local deformation is assumed to act at $\mathcal{B}$ 's endpoint of the overlap segment $x_{i}$ in the direction of the overlap segment. The normal component of the $i$ th contact force $f_{i}^{n}$ is assumed to depend on $\delta_{i}^{n}$ in terms of a function $g_{i}\left(\delta_{i}^{n}\right)$. This function is required to be differentiable, zero when $\delta_{i}^{n}$ is zero, and monotonically increasing when $\delta_{i}^{n}$ is positive. Thus

$$
f_{i}^{n}=g_{i}\left(\delta_{i}^{n}\right), \text { such that } g_{i}^{\prime}\left(\delta_{i}^{n}\right)>0 \text { when } \delta_{i}^{n}>0 \text {. }
$$

It is important to note that a wide variety of contact models can be represented in this framework. The simplest contact model assumes that $g_{i}$ is a linear function of the overlap $g_{i}\left(\delta_{i}^{n}\right)=$ $\kappa_{i} \delta_{i}^{n}$, where the coefficient $\kappa_{i}$ represents the combined stiffness of $\mathcal{B}$ and $\mathcal{A}_{i}$ at the contact [13]. The Hertz model [12], which has been verified theoretically and experimentally, establishes that $g_{i}\left(\delta_{i}^{n}\right)=\kappa_{i}\left(\delta_{i}^{n}\right)^{3 / 2}$ where $\kappa_{i}$ is a specific function of the bodies' local material and geometric properties. The overlap representation (1) is valid even under more general circumstances than those assumed by the Hertz model. For instance, soft fingertips generate contact areas which are not necessarily small compared with the bodies' size, yet the resultant normal force can still be expressed as a function of the overlap [41], [42]. With this background, the principle of micro-penetration is as follows.

Lemma 2.1 (Micro-Penetration): Let an object $\mathcal{B}$ contact a stationary finger $\mathcal{A}_{i}$, such that both bodies are quasi-rigid. The change in the normal component of the $i$ th contact force due to an instantaneous motion $\dot{q}$ of $\mathcal{B}$ is

$$
\Delta f_{i}^{n}=-\sigma_{i}^{n}\left(n_{i} \cdot \dot{X}_{r_{i}}\right)=-\sigma_{i}^{n}\left(n_{i} \cdot G_{i}^{T} \dot{q}\right), \text { for some } \sigma_{i}^{n}>0
$$

${ }^{3}$ The notion of overlap used here is consistent with the concept of "relative approach" in the contact mechanics literature. 
where $n_{i}$ is the inward pointing unit normal to $\mathcal{B}$ at $x_{i}$, and $\sigma_{i}^{n}$ is a positive multiple of $g_{i}^{\prime}\left(\delta_{i}^{n}\right)$. The interpretation of (2) is as follows. The vector $-n_{i}$ points outward with respect to $\mathcal{B}$. If $\left(-n_{i}\right) \cdot \dot{X}_{r_{i}}>0$, the overlap between $\mathcal{B}$ and the stationary finger $\mathcal{A}_{i}$ increases to first-order, due to the instantaneous motion of $\mathcal{B}$, and consequently $\Delta f_{i}^{n}>0$. The converse holds when $\left(-n_{i}\right)$. $\dot{X}_{r_{i}}<0$, since in this case, the overlap between the two bodies is decreasing.

Proof: Let $q_{0}$ be the initial preload configuration of $\mathcal{B}$, and let $q(t)$ be a c-space trajectory of $\mathcal{B}$ such that $q(0)=q_{0}$ and $\left.(d / d t)\right|_{t=0} q(t)=\dot{q}$. Then a small change in $f_{i}^{n}$ along $q(t)$ can be written as $\Delta f_{i}^{n}=(d / d t)_{t=0}$ $f_{i}^{n}(q(t)) t+o\left(t^{2}\right)$, where $t \geq 0$. Using the chain rule on (1), $(d / d t) f_{i}^{n}(q(t))=g_{i}^{\prime}\left(\delta_{i}^{n}\right)(d / d t) \delta_{i}^{n}(q(t))$. According to Lemma $A .2$ in the Appendix, $(d / d t) \delta_{i}^{n}(q(t))=-n_{i} \cdot \dot{X}_{r_{i}}$. Hence, $\Delta f_{i}^{n}=-\sigma_{i}^{n}\left(n_{i} \cdot \dot{X}_{r_{i}}\right)$, where $\sigma_{i}^{n}=g_{i}^{\prime}\left(\delta_{i}^{n}\right) t>0$ by the monotonicity of $g_{i}$ with respect to $\delta_{i}^{n}$.

The lemma can be written in matrix form as the following principle of micro-penetration:

$$
\begin{aligned}
\left(\begin{array}{c}
\Delta f_{1}^{n} \\
\vdots \\
\Delta f_{k}^{n}
\end{array}\right) & =-\left[\begin{array}{ccc}
\sigma_{1}^{n} & & \\
& \ddots & \\
& & \sigma_{k}^{n}
\end{array}\right] N\left[G_{1} \cdots G_{k}\right]^{T} \dot{q} \\
& =-\Sigma_{n} N G^{T} \dot{q}, \quad \text { for some } \sigma_{1}^{n}, \ldots, \sigma_{k}^{n}>0
\end{aligned}
$$

where $\Sigma_{n}=\operatorname{diag}\left(\sigma_{1}^{n}, \ldots, \sigma_{k}^{n}\right), N$ is the normal projection matrix and $G=\left[G_{1} \ldots G_{k}\right]$ is the grasp matrix. Note that (3) represents a coupling of the changes in the normal component of the contact forces through the instantaneous motion $\dot{q}$ of $\mathcal{B}$. This is nothing more than a general linearized stiffness relationship that holds for any quasi-rigid-body contact compliance model. Note, too, that (3) merely states that the coefficients $\sigma_{1}^{n}, \ldots, \sigma_{k}^{n}$ have some unspecified positive value. The ensuing bound on the indeterminate contact forces is based only on this limited information and not on any specific value for these coefficients.

\section{Mechanics of Micro-Slip}

Next, we formulate a generic rule for the change in the tangential or frictional component of a contact force due to material deformation at a quasi-rigid contact. This rule covers most compliance models that have been proposed in the literature, and is, therefore, quite general.

The principle of micro-slip is based on tangential compliance induced at a frictional contact by local material deformation. The process underlying this phenomenon is as follows. When two quasi-rigid bodies are preloaded along the normal direction, they locally deform to establish a contact patch centered at the original contact point [see Fig. 3(a)]. The deformed bodies generate a normal force field which is continuously distributed along the contact patch. (The integral of this force field over the contact area gives the net normal force.) When the two bodies are next loaded along a tangential direction, they locally deform in a way that generates a tangential force field which is continuously distributed along the contact patch [see Fig. 3(b)]. The usual assumption made in the solid mechanics literature is that the normal and tangential force fields interact at the individual points of the contact area according to Coulomb's law. Under this assumption, elasticity theory as well as experimental
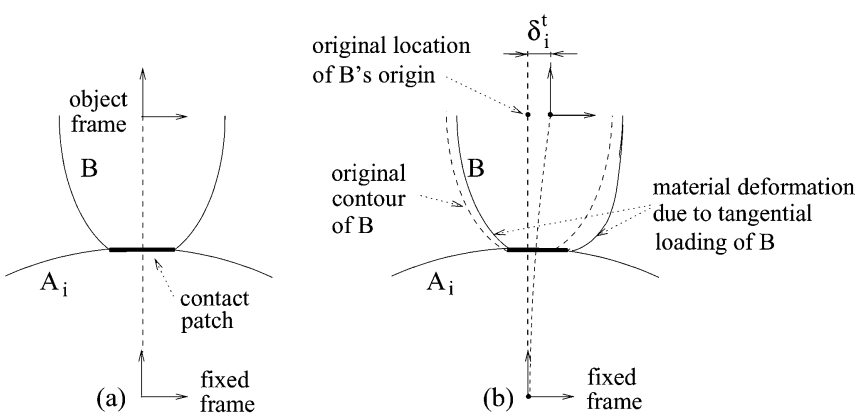

Fig. 3. (a) Initial contact patch is generated by normal loading of $\mathcal{B}$ against $\mathcal{A}_{i}$. (b) Tangential loading of $\mathcal{B}$ causes tangential displacement of $\mathcal{B}$ without any macro-slip.

measurements indicate that micro-slip takes place in an outer region of the contact area. However, an inner disc of the contact area remains stationary. As the magnitude of the tangential loading increases the area of the inner disc shrinks, but the two bodies do not experience any macro-slip, since there is still a stationary inner disc. Only when the net tangential loading reaches $\mu$ times the net normal loading ( $\mu$ being the coefficient of friction), the inner disc shrinks to a point and the two bodies experience macro-slip at the contact.

The sequential normal and tangential loading of a contact is the first loading profile that has been investigated in the literature by Mindlin and Deresiewicz [22]. Subsequent work by Walton applied similar principles to a linear loading profile, where a fixed ratio between tangential and normal displacements is kept during the loading process [37]. This loading profile generates a tangential compliance law which involves no micro-slip. However, Elata and Berryman demonstrated that when a linear loading is followed by a linear unloading of a different slope, micro-slip occurs along an outer ring of the contact area [10]. For our purposes, we only need the basic fact that material deformation at a quasi-rigid contact induces nonlinear tangential compliance, with certain generic properties which are common to the various loading profiles.

We now formulate a rule for the change in the tangential component of the contact force, assuming that the contacting bodies deform but do not slip. In our case, the finger $\mathcal{A}_{i}$ is stationary, while $\mathcal{B}$ moves along a c-space trajectory $q(t)$. Let $\delta_{i}^{t}(q(t))$ denote the tangential displacement of $\mathcal{B}$ relative to the $i$ th contact due to motion of $\mathcal{B}$. Then, the derivative of $\delta_{i}^{t}$ along $q(t)$ is given by projection of the velocity of the overlap-segment endpoint $x_{i}$ along the unit tangent $t_{i}$ as

$$
\frac{d}{d t} \delta_{i}^{t}(q(t))=t_{i} \cdot \dot{X}_{r_{i}}=t_{i} \cdot G_{i}^{T} \dot{q}(t) .
$$

Note that in contrast with $\delta_{i}^{n}(q)$, the tangential displacement is not a direct function of $q$, but requires integration of (4) over the entire loading trajectory $[15$, p. 221]. The net tangential force opposes the direction of tangential displacement. The tangential component of the $i$ th contact force $f_{i}^{t}$ has a dominantly elastic nature which obeys a law of the general form

$$
f_{i}^{t}=-h_{i}\left(\delta_{i}^{t}, \delta_{i}^{n}\right) \text { as long as } f_{i}^{n}>0 \text { and }\left|f_{i}^{t}\right| \leq \mu f_{i}^{n} .
$$

(The tangential force-displacement relationship has an additional small dissipative component which is caused by 
micro-slip in an outer region of the contact area. This dissipation further opposes the motion and does not affect the generic rule derived below.) The function $h_{i}$ has the following properties. It is differentiable, $h_{i}\left(0, \delta_{i}^{n}\right)=0$, and for any fixed positive $\delta_{i}^{n}$, it is monotonically increasing in $\delta_{i}^{t}$. The monotonicity of $h_{i}$ implies that the tangential force in (5) opposes the direction of tangential displacement. Note that $f_{i}^{t}$ depends both on the tangential displacement $\delta_{i}^{t}$ and the normal penetration $\delta_{i}^{n}$. However, in practice, the variation of $h_{i}$ with respect to $\delta_{i}^{n}$ is significantly lower than the variation with respect to $\delta_{i}^{t}$. Hence, we make a simplifying assumption that $h_{i}$ is approximately $h_{i}\left(\delta_{i}^{t}, \delta_{i}^{n}\left(q_{0}\right)\right)$, where $\delta_{i}^{n}\left(q_{0}\right)$ is the normal penetration at the preload configuration $q_{0}$. This assumption allows a simple characterization of the polyhedral bound. ${ }^{4}$

The following lemma summarizes the rule for the change in the tangential component of the contact force.

Lemma 2.2 (Micro-Slip): Let an object $\mathcal{B}$ be in contact with a stationary finger $\mathcal{A}_{i}$, such that the two bodies are quasi-rigid. Let the tangent and normal components of the $i$ th contact force satisfy $\left|f_{i}^{t}\right| \leq \mu f_{i}^{n}$ where $f_{i}^{n}>0$. Then the change in the tangential component of the $i$ th contact force due to an instantaneous motion $\dot{q}$ of $\mathcal{B}$ is

$$
\Delta f_{i}^{t}=-\sigma_{i}^{t}\left(t_{i} \cdot \dot{X}_{r_{i}}\right)=-\sigma_{i}^{t}\left(t_{i} \cdot G_{i}^{T} \dot{q}\right), \text { for some } \sigma_{i}^{t}>0
$$

where $t_{i}$ is the unit tangent to $\mathcal{B}$ at $x_{i}$, and $\sigma_{i}^{t}$ is a positive multiple of $\partial h_{i}\left(\delta_{i}^{t}, \delta_{i}^{n}\right) / \partial \delta_{i}^{t}$.

Proof: The proof is similar to the proof of Lemma 2.1. First, one applies the chain rule $(d / d t) f_{i}^{t}(q(t))=$ $-h_{i}^{\prime}\left(\delta_{i}^{t}, \delta_{i}^{n}\right)(d / d t) \delta_{i}^{t}(q(t))$, where $h_{i}^{\prime}$ denotes derivative with respect to $\delta_{i}^{t}$. Then, one uses (4) to obtain $\Delta f_{i}^{t}=-\sigma_{i}^{t}\left(t_{i} \cdot \dot{X}_{r_{i}}\right)$, where $\sigma_{i}^{t}=t \cdot h_{i}^{\prime}\left(\delta_{i}^{t}, \delta_{i}^{n}\right)>0$ by the monotonicity of $h_{i}$ with respect to $\delta_{i}^{t}$.

The lemma simply states that $\Delta f_{i}^{t}$ is proportional to the tangential displacement of $\mathcal{B}$ induced by $\dot{q}$, with the sign of $\Delta f_{i}^{t}$ opposing the direction of tangential displacement. We can write (6) in matrix form as the following principle of micro-slip:

$$
\begin{aligned}
\left(\begin{array}{c}
\Delta f_{1}^{t} \\
\vdots \\
\Delta f_{k}^{t}
\end{array}\right) & =-\left[\begin{array}{ccc}
\sigma_{1}^{t} & & \\
& \ddots & \\
& & \sigma_{k}^{t}
\end{array}\right] T\left[G_{1} \cdots G_{k}\right]^{T} \dot{q} \\
& =-\Sigma_{t} T G^{T} \dot{q}, \text { for some } \sigma_{1}^{t}, \ldots, \sigma_{k}^{t}>0
\end{aligned}
$$

where $\Sigma_{t}=\operatorname{diag}\left(\sigma_{1}^{t}, \ldots, \sigma_{k}^{t}\right), T$ is the tangent projection matrix, and $G$ is the grasp matrix. Much like the micro-penetration principle discussed above, the micro-slip principle represents a coupling of the changes in the tangential component of the contact forces through the instantaneous motion $\dot{q}$ of $\mathcal{B}$. Moreover, this coupling is based on the limited information that the coefficients $\sigma_{1}^{t}, \ldots, \sigma_{k}^{t}$ have some unspecified positive value.

In summary, the two quasi-rigid modeling principles represent generic relationships between body movement and finger reaction forces. Specific information about the contact parameters is not needed, and the principles are valid for a wide range of different compliance models.

\footnotetext{
${ }^{4}$ This assumption can be removed, with the result that the indeterminate contact forces are still bounded in a polyhedral set, albeit one with a more complicated geometry.
}

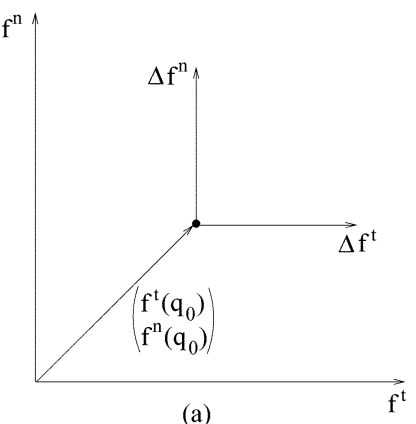

(a)

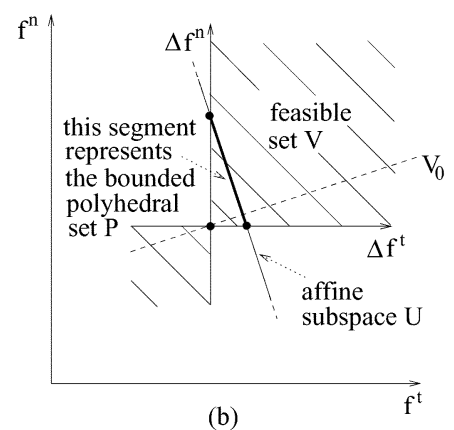

(b)
Fig. 4. (a) Origin of $\Delta f$-space is determined by the preload forces. (b) Feasible set $V$ fills entire quadrants in $\Delta f$-space.

\section{Derivation of the Polyhedral Bound}

We now derive the polyhedral bound on the indeterminate contact forces. First, let us clarify our objective. A planar object $\mathcal{B}$ is initially held in a preloaded equilibrium grasp at a configuration $q_{0}$. When an external wrench $\boldsymbol{w}_{\text {ext }}$ acts on $\mathcal{B}$, we assume that none of the contacts breaks or slips in response to $w_{\text {ext }}$. Our objective is to provide a polyhedral bound on the possible contact-force changes at the new equilibrium induced by $\boldsymbol{w}_{\text {ext }}$. Next, we introduce some notation. The coordinates of contact force space are denoted collectively as $\left(f^{t}, f^{n}\right) \in \mathbb{R}^{2 k}$, where $f^{t}=\left(f_{1}^{t}, \ldots, f_{k}^{t}\right)$ and $f^{n}=\left(f_{1}^{n}, \ldots, f_{k}^{n}\right)$. The components of the preload forces are denoted $\left(f^{t}\left(q_{0}\right), f^{n}\left(q_{0}\right)\right) \in$ $\mathbb{R}^{2 k}$. The changes in the contact forces induced by $\boldsymbol{w}_{\text {ext }}$ are denoted $\left(\Delta f^{t}, \Delta f^{n}\right)$, where $\Delta f^{t}=\left(\Delta f_{1}^{t}, \ldots, \Delta f_{k}^{t}\right)$ and $\Delta f^{n}=$ $\left(\Delta f_{1}^{n}, \ldots, \Delta f_{k}^{n}\right)$. These changes lie in a linear space called $\Delta f$-space, which is a copy of $\mathbb{R}^{2 k}$ based at the preload point $\left(f^{t}\left(q_{0}\right), f^{n}\left(q_{0}\right)\right)$ [see Fig. 4(a)].

The grasp matrix $G$ maps the contact forces to the net wrench acting on $\mathcal{B}$. We begin by writing $G$ as a linear mapping, denoted $\bar{G}$, from contact force space to the object's wrench space. The contact forces are given in terms of their tangent and normal components by the formula $f=T^{T} f^{t}+N^{T} f^{n}$, where $T$ and $N$ are the $k \times 2 k$ tangent and normal projection matrices. Premultiplying both sides by $G$ gives

$$
\begin{aligned}
G \boldsymbol{f}=G\left[\begin{array}{l}
T \\
N
\end{array}\right]^{T}\left(\begin{array}{l}
f^{t} \\
f^{n}
\end{array}\right)=\bar{G}\left(\begin{array}{l}
f^{t} \\
f^{n}
\end{array}\right), \\
\text { where } \bar{G} \triangleq G\left[\begin{array}{c}
T \\
N
\end{array}\right]^{T}=3 \times 2 k .
\end{aligned}
$$

From now on, we refer to $\bar{G}$ as the grasp matrix.

Next, we write two key expressions involving the matrix $\bar{G}$. The preload forces at the initial equilibrium grasp satisfy

$$
\bar{G}\left(\begin{array}{c}
f^{t}\left(q_{0}\right) \\
f^{n}\left(q_{0}\right)
\end{array}\right)=\overrightarrow{0}
$$

The first key expression is the condition for equilibrium induced by an external wrench $\boldsymbol{w}_{\text {ext }}$ acting on $\mathcal{B}$. The object and surrounding fingers respond to $w_{\text {ext }}$ by locally deforming at the contacts. The new forces $\left(f^{t}, f^{n}\right)=\left(f^{t}\left(q_{0}\right), f^{n}\left(q_{0}\right)\right)+$ $\left(\Delta f^{t}, \Delta f^{n}\right)$ form an equilibrium with $\boldsymbol{w}_{\text {ext }}$ according to the linear inhomogeneous equation

$$
\bar{G}\left(\begin{array}{c}
f^{t} \\
f^{n}
\end{array}\right)+\boldsymbol{w}_{\mathrm{ext}}=\bar{G}\left(\begin{array}{c}
\Delta f^{t} \\
\Delta f^{n}
\end{array}\right)+\boldsymbol{w}_{\mathrm{ext}}=\overrightarrow{0}
$$


where we have used (8) to eliminate the preload forces $\left(f^{t}\left(q_{0}\right), f^{n}\left(q_{0}\right)\right)$. The set of all solutions $\left(\Delta f^{t}, \Delta f^{n}\right)$ to (9) forms an affine subspace denoted $U$ [see Fig. 4(b)]. Next, we introduce a parametrization for $U$ in terms of the null space of $\bar{G}$. Let $m$ be the dimension of the kernel of $\bar{G}$, where $m=2 k-3$ in the generic case where $\bar{G}$ has full rank. Let $E$ be the $2 k \times m$ matrix whose columns span the kernel of $\bar{G}$, i.e., $\bar{G} E=\overrightarrow{0}$. We use in the parametrization of $U$ the particular solution of (9) given by $\left(\Delta f^{t}, \Delta f^{n}\right)=-\bar{G}^{\dagger} \boldsymbol{w}_{\text {ext }}$, where $\bar{G}^{\dagger}=\bar{G}^{T}\left[\bar{G} \bar{G}^{T}\right]^{-1}$ is the $2 k \times 3$ pseudoinverse of $\bar{G}$. Using this particular solution, $U$ is parametrized by

$$
U=\left\{\left(\begin{array}{c}
\Delta f^{t} \\
\Delta f^{n}
\end{array}\right)=E \boldsymbol{\nu}-\bar{G}^{\dagger} w_{\mathrm{ext}}: \boldsymbol{\nu} \in \mathbb{R}^{m}\right\}
$$

The second key expression specifies what changes in the contact forces are generated by instantaneous motions of $\mathcal{B}$, while the fingers remain stationary. We call the collection $\left(\Delta f^{t}, \Delta f^{n}\right)$ generated by all instantaneous motions of $\mathcal{B}$ the feasible set $V$. Using the micro-penetration and micro-slip principles from the previous section, the contact force changes induced by $\dot{q}$ are

$$
\left(\begin{array}{c}
\Delta f^{t} \\
\Delta f^{n}
\end{array}\right)=-\left(\begin{array}{c}
\Sigma_{t} T G^{T} \dot{q} \\
\Sigma_{n} N G^{T} \dot{q}
\end{array}\right)=-\left[\begin{array}{cc}
\Sigma_{t} & 0 \\
0 & \Sigma_{n}
\end{array}\right]\left[\begin{array}{c}
T \\
N
\end{array}\right] G^{T} \dot{q}
$$

Since $\bar{G}=G\left[\begin{array}{l}T \\ N\end{array}\right]^{T}$, the feasible set is given by

$$
V=\left\{\left(\begin{array}{c}
\Delta f^{t} \\
\Delta f^{n}
\end{array}\right)=-\Sigma \bar{G}^{T} \dot{q}: \dot{q} \in \mathbb{R}^{3} \text { and } \Sigma>0\right\}
$$

where $\Sigma \triangleq \operatorname{diag}\left(\Sigma_{t}, \Sigma_{n}\right)$ contains the unspecified positive parameters $\sigma_{1}^{t}, \ldots, \sigma_{k}^{t}$ and $\sigma_{1}^{n}, \ldots, \sigma_{k}^{n}$. In the generic case where $\bar{G}$ has full rank, $V$ is a collection of three-dimensional (3-D) subspaces in $\Delta f$-space, each corresponding to a particular value of $\Sigma$. The set of contact force changes due to $\boldsymbol{w}_{\text {ext }}$, denoted $\mathcal{P}$, is given by $\mathcal{P}=U \cap V$. Once $\mathcal{P}$ is known, the statically feasible contact forces induced by $\boldsymbol{w}_{\text {ext }}$ are given by $\left(f^{t}, f^{n}\right)=$ $\left(f^{t}\left(q_{0}\right), f^{n}\left(q_{0}\right)\right)+\left(\Delta f^{t}, \Delta f^{n}\right)$ such that $\left(\Delta f^{t}, \Delta f^{n}\right) \in \mathcal{P}$.

We now show that $\mathcal{P}$ is a bounded polyhedral set in $\Delta f$-space. We first argue that $\mathcal{P}$ is polyhedral and then demonstrate its boundedness. Let a $p$-quadrant in $\mathbb{R}^{p}$ be the rectangular cone spanned by a particular choice of directions along the coordinate axes of $\mathbb{R}^{p}$ (there are $2^{p}$ such $p$-quadrants in $\mathbb{R}^{p}$ ). According to Lemma B.1 in the Appendix, the feasible set $V$ consists of entire $2 k$-quadrants in $\Delta f$-space [see Fig. $4($ b) ]. Hence, $V$ is an unbounded polyhedral set. On the other hand, $U$ is an affine subspace in $\left(\Delta f^{t}, \Delta f^{n}\right)$-space. Combining these two facts, the intersection $\mathcal{P}$ is a polyhedral set. However, it still remains to show that $\mathcal{P}$ is bounded. The next proposition is a key result of the paper.

Proposition 3.1 (Boundedness): The polyhedral set of contact-force changes induced by the action of $\boldsymbol{w}_{\text {ext }}$ on $\mathcal{B}, \mathcal{P}=$ $\mathcal{U} \cap \mathcal{V}$ is bounded in $\Delta f$-space.

The intuition behind the boundedness of $\mathcal{P}$ is as follows. Let the skeletal set of $V$ be the subset

$$
V_{0} \triangleq\left\{\left(\begin{array}{c}
\Delta f^{t} \\
\Delta f^{n}
\end{array}\right)=\bar{G}^{T} \dot{q}: \dot{q} \in T_{q_{0}} \mathbb{R}^{3}\right\} .
$$

It is shown in Lemma B.l in the Appendix that the feasible set $V$ consists of the $2 k$-quadrants occupied by $V_{0}$. On the other hand, $V_{0}$ is spanned by the rows of $\bar{G}$. Hence, $V_{0}$ is orthogonal to the kernel of $\bar{G}$ as well as to the affine subspace $U$ [Fig. 4(b)]. It follows that $U$ is transversal to the $2 k$-quadrants occupied by $V$, and consequently, $\mathcal{P}=U \cap V$ must be bounded. The following proof is based on an argument presented in a 1964 paper by Ben-Israel [1].

Proof: According to Lemma B.1, the feasible set $V$ fills the $2 k$-quadrants in $\Delta f$-space whose interior is occupied by the skeletal set $V_{0}$. Let $\mathcal{I}$ denote the index set of the $2 k$-quadrants $K_{i}$ occupied by $V$. Then, $\mathcal{P}$ is the union $\mathcal{P}=U \cap V=\cup_{i \in \mathcal{I}} U \cap K_{i}$. Hence, $\mathcal{P}$ is bounded if each piece $U \cap K_{i}(i \in \mathcal{I})$ is either empty or bounded. Thus, consider a particular $2 k$-quadrant $K_{i}$ such that $U \cap K_{i}$ is nonempty. Let $z=\left(z_{1}, \ldots, z_{2 k}\right)$ be a unit vector in $V_{0}$ which also lies in the interior of $K_{i}$. Since the $2 k$ entries of $z$ are nonzero, $m=\min _{j=1, \ldots, 2 k}\left\{\left|z_{j}\right|\right\}$ is strictly positive. Let $y=\left(y_{1}, \ldots, y_{2 k}\right)$ be any vector from $U \cap K_{i}$. Since $y \in U$, it can be written as $y=E \boldsymbol{\nu}-\bar{G}^{\dagger} \boldsymbol{w}_{\text {ext }}$ for some $\boldsymbol{\nu} \in \mathbb{R}^{m}$. Since $y$ lies in the same $2 k$-quadrant as $z$, it satisfies $y_{j} z_{j} \geq 0$ for $j=1, \ldots, 2 k$. It follows that each $y_{j} z_{j}$ satisfies the inequality

$$
0 \leq y_{j} z_{j} \leq y \cdot z=\left(E \boldsymbol{\nu}-\bar{G}^{\dagger} \boldsymbol{w}_{\text {ext }}\right) \cdot z \leq\left\|\bar{G}^{\dagger} \boldsymbol{w}_{\text {ext }}\right\|
$$

where we used the fact that $z \in V_{0}$ is orthogonal to the kernel of $\bar{G}$. Since $\left|z_{j}\right| \geq m>0$, we obtain the bound $\left|y_{j}\right| \leq\left\|\bar{G}^{\dagger} \boldsymbol{w}_{\text {ext }}\right\| / m$ for $j=1, \ldots, 2 k$. The norm of $y$ is therefore bounded by $\|y\| \leq \sqrt{2 k}\left\|\bar{G}^{\dagger} \boldsymbol{w}_{\text {ext }}\right\| / m$, which is a finite constant.

Let us pause to discuss a physical interpretation of the orthogonality between the skeletal set $V_{0}$ and the affine subspace $U$. Multiplying the representation (9) of $U$ with $\dot{q}$ gives

$$
\left(\begin{array}{c}
\Delta f^{t} \\
\Delta f^{n}
\end{array}\right) \cdot \bar{G}^{T} \dot{q}+w_{\text {ext }} \cdot \dot{q}=0 \text { for all }\left(\begin{array}{c}
\Delta f^{t} \\
\Delta f^{n}
\end{array}\right) \in U \text { and } \dot{q} \in \mathbb{R}^{3} .
$$

Since $V_{0}$ consists of vectors of the form $\bar{G}^{T} \dot{q}$, (12) represents the orthogonality between $V_{0}$ and $U$. However, the entries of $\bar{G}^{T} \dot{q}$ are the contact-point velocities $\dot{X}_{r_{1}}, \ldots, \dot{X}_{r_{k}}$ induced by $\dot{q}$. The entries of $\left(\Delta f^{t}, \Delta f^{n}\right) \in U$ are the contact-force changes induced by $\boldsymbol{w}_{\text {ext }}$. Letting $\Delta \boldsymbol{f}_{1}, \ldots, \Delta \boldsymbol{f}_{k}$ denote these changes, the orthogonality between $V_{0}$ and $U$ asserts that $\sum_{i=1}^{k} \Delta \boldsymbol{f}_{i}$. $\dot{X}_{r_{i}}+\boldsymbol{w}_{\text {ext }} \cdot \dot{q}=0$. In other words, the energy flowing into the grasping system through the action of $\boldsymbol{w}_{\text {ext }}$ on $\mathcal{B}$ is stored as an elastic energy associated with local deformation at the contacts. ${ }^{5}$

The following theorem summarizes the results concerning the indeterminate contact forces.

Theorem 1: Let a planar object $\mathcal{B}$ be held in an equilibrium grasp by $k$ fingers with preload forces $\left(f^{t}\left(q_{0}\right), f^{n}\left(q_{0}\right)\right)$. Let an external wrench $\boldsymbol{w}_{\text {ext }}$ act on $\mathcal{B}$, such that none of the contacts breaks or slips. Then, the statically feasible contact forces induced by $\boldsymbol{w}_{\text {ext }}$ are given by

$$
\left(\begin{array}{l}
f^{t} \\
f^{n}
\end{array}\right)=\left(\begin{array}{c}
f^{t}\left(q_{0}\right) \\
f^{n}\left(q_{0}\right)
\end{array}\right)+\left(\begin{array}{c}
\Delta f^{t} \\
\Delta f^{n}
\end{array}\right) \text { such that }\left(\begin{array}{c}
\Delta f^{t} \\
\Delta f^{n}
\end{array}\right) \in \mathcal{P}
$$

\footnotetext{
5Physically, some energy is being lost due to micro-slip at the contacts, but this loss is relatively small in the quasi-static setting considered here.
} 
where $\mathcal{P}$ is a bounded $\boldsymbol{m}$-dimensional polyhedral set, and generically $m=2 k-3$.

Several comments are in order here. First, the polyhedral bound does not require any specific knowledge of the contact parameters $\sigma_{1}^{t}, \ldots, \sigma_{k}^{t}$ and $\sigma_{1}^{n}, \ldots, \sigma_{k}^{n}$. The bound is thus useful for robust grasp synthesis, as discussed below. However, while the bound reduces the unbounded linear space of indeterminate contact forces to a bounded polyhedral subset, its dimension is generically the same as the dimension of the original linear space. Special cases where the polyhedral set has a smaller dimension occur when the kernel of $\bar{G}$ consists of pure normal forces or pure tangential forces, as illustrated in Example 2 below. Second, any knowledge of specific intervals where the contact parameters can vary would only improve the bound, shrinking $\mathcal{P}$ to a smaller polyhedral set. This fact is stated in the following corollary.

Corollary 3.2: Let the contact parameters vary in intervals $\sigma_{\min }^{t} \leq \sigma_{1}^{t}, \ldots, \sigma_{k}^{t} \leq \sigma_{\max }^{t}$ and $\sigma_{\min }^{n} \leq \sigma_{1}^{n}, \ldots, \sigma_{k}^{n} \leq \sigma_{\max }^{n}$, such that $0 \leq \sigma_{\min }^{t}<\sigma_{\max }^{t} \leq \infty$ and $0 \leq \sigma_{\min }^{n}<\sigma_{\max }^{n} \leq \infty$. Then, $\mathcal{P}$ based on these intervals is still a bounded polyhedral set in $\Delta f$-space. Moreover, if a polyhedral set $\mathcal{P}_{1}$ is obtained with contact parameters varying in subintervals of the intervals used to compute another polyhedral set $\mathcal{P}_{2}$, then $\mathcal{P}_{1} \subseteq \mathcal{P}_{2}$.

A proof of the corollary is relegated to [34]. Third, the theorem assumes that none of the contacts break or slip in response to $\boldsymbol{w}_{\text {ext }}$. In order to satisfy this assumption, one must select contact locations and preload forces such that the friction-cone constraints are satisfied for the entire polyhedral set $\mathcal{P}$. We postpone this operation to the last section discussing grasp synthesis.

The remainder of this section contains three results that lead to a polynomial time algorithm for computing $\mathcal{P}$. The first result asserts that the polyhedral bound is always nonempty and connected.

Lemma 3.3: The polyhedral set $\mathcal{P}$ consists of a single connected component in $\Delta f$-space. Moreover, this set is always nonempty and contains the solution $-\bar{G}^{\dagger} \boldsymbol{w}_{\text {ext }}$.

A proof of the lemma appears in Appendix B. Note that $P=$ $U \cap V$ always contains the solution $-\bar{G}^{\dagger} \boldsymbol{w}_{\text {ext }}$, since it belongs to $U$ by setting $\boldsymbol{\nu}=0$ in (10), and it belongs to $V$ by setting $\Sigma=I$ and $\dot{q}=\left[\bar{G} \bar{G}^{T}\right]^{-1} \boldsymbol{w}_{\text {ext }}$ in (11). The lemma implies that the indeterminate contact forces, which are obtained by adding the preload forces to the set of contact-force changes, form a nonempty and connected polyhedral set. In practice, one has to verify conditions such as friction-cone constraints over the entire set of possible contact forces, and having to deal with a single connected component rather than a collection of disjoint sets greatly simplifies this task. The second result characterizes the planar facets of $\mathcal{P}$.

Lemma 3.4: The planar facets of the polyhedral set $\mathcal{P}$ are embedded in the coordinate hyperplane of $\left(\Delta f^{t}, \Delta f^{n}\right)$-space. Moreover, the $i$ th facet of $\mathcal{P}$ satisfies the formula

$$
\boldsymbol{e}_{\boldsymbol{i}} \cdot \boldsymbol{\nu}-\left(\bar{G}^{\dagger} \boldsymbol{w}_{\text {ext }}\right)_{i}=0, \text { where } \boldsymbol{\nu} \in \mathbb{R}^{m}
$$

where $\boldsymbol{e}_{i} \in \mathbb{R}^{m}$ is the $i$ th row of $E$, and $\left(\bar{G}^{\dagger} \boldsymbol{w}_{\text {ext }}\right)_{i}$ is the $i$ th component of $\bar{G}^{\dagger} \boldsymbol{w}_{\text {ext }} \in \mathbb{R}^{2 k}$.

A proof of the lemma also appears in Appendix B. The lemma asserts that each planar facet of $\mathcal{P}$ is associated with the vanishing of a tangent or normal force component at one of the
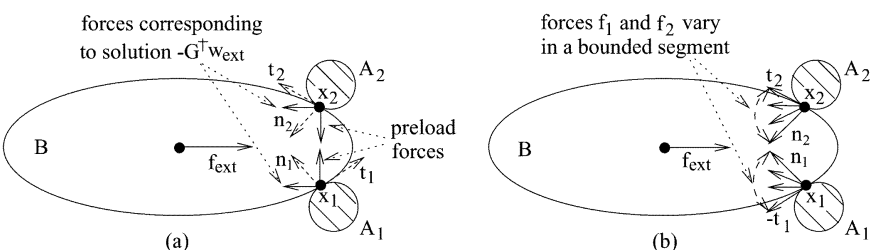

Fig. 5. Two-finger grasp of an ellipse. (a) Preload forces and solution $-\bar{G}^{\dagger} \boldsymbol{w}_{\text {ext }}$. (b) Forces of the bounded segment $\mathcal{P}$.

contacts. This insight is used below to graphically depict the polyhedral bound in certain simple grasp arrangements. In more complex grasps, one must use the algorithm described in the Appendix. The algorithm is based on the following characterization of the vertices and convex cells of $\mathcal{P}$.

Lemma 3.5: The set $\mathcal{P}$ is a union of convex polyhedra in $\Delta f$-space. The number of convex polyhedra is bounded from above by $(2 k)^{2}$, and the total number of vertices is bounded from above by $(2 k)^{3}$, where $k$ is the number of contacts.

A proof of the lemma appears in Appendix B. The convex polyhedra mentioned in the lemma are the pieces of $\mathcal{P}$ contained in the individual $2 k$-quadrants of $\Delta f$-space. The bound on the number of convex pieces is merely a bound on the number of $2 k$-quadrants occupied by the feasible set $V$. Since $\mathcal{P}$ is the intersection of $V$ with the affine subspace $U$, the actual number of convex pieces in $\mathcal{P}$ is usually smaller. As an illustration of this fact, let us compare the two bounds with the actual numbers obtained in Example 3 below, which computes the set $\mathcal{P}$ for a particular three-finger grasp arrangement. First, the more precise bound on the number of $2 k$-quadrants occupied by $V$ is $2 k(2 k-1)+2$ (see the proof). Substituting $k=3$ gives $2 k(2 k-1)+2=32$, and, indeed, $V$ occupies 24 out of the 64 6-D quadrants in Example 3. Second, the more exact bound on the number of vertices in $\mathcal{P}$ is $(1 / 6) 2 k(2 k-1)(2 k-2)$ (see proof). Substituting $k=3$ gives $(1 / 6) 2 k(2 k-1)(2 k-2)=20$, and, indeed, $\mathcal{P}$ has 18 vertices in Example 3 . The lemma leads to a polynomial time algorithm for computing $\mathcal{P}$. The algorithm, described in Appendix C, accepts as input the grasp matrix $\bar{G}$ and an external wrench $\boldsymbol{w}_{\text {ext }}$. The algorithm first determines which $2 k$-quadrants of $\Delta f$-space are occupied by the feasible set $V$. Then, it determines which of these $2 k$-quadrants is also intersected by the affine subspace $U$. Finally, the algorithm constructs as its output lists of inequalities describing the convex pieces of $\mathcal{P}$. The details of the algorithm and its time complexity are discussed in Appendix C.

\section{EXAMPLES OF THE POLYHEDRAL BOUND}

This section contains three detailed examples of the polyhedral bound. We describe the bound under the assumption that none of the contacts breaks or slips in response to the applied wrench. This assumption is considered in the next section discussing grasp synthesis.

Example 1: The first example, shown in Fig. 5(a), is an ellipse held by two disc fingers in a frictional grasp. The ellipse is subjected to a horizontal external force $\boldsymbol{f}_{\text {ext }}$ that acts at the ellipse's center against the fingers. The grasp matrix $\bar{G}$ is $3 \times 4$, and it has a 1-D kernel. It follows from Theorem 1 that $\mathcal{P}$ is a bounded segment, and we now determine this segment. Re- 


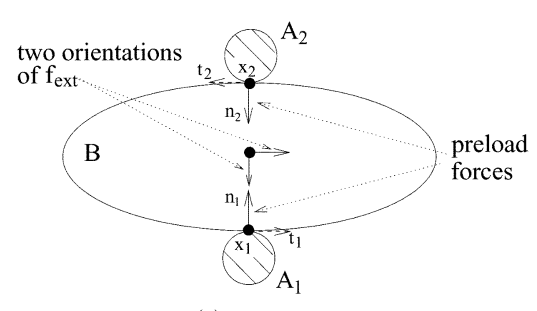

(a)

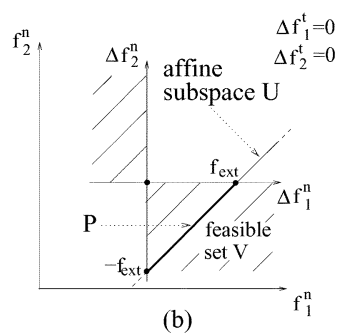

(b)

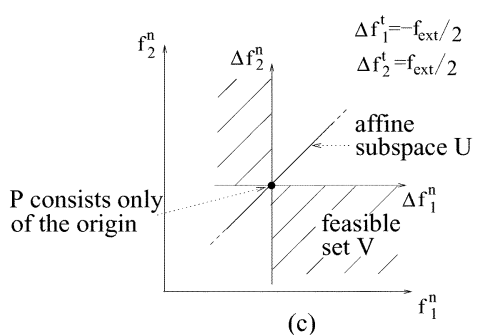

(c)

Fig. 6. (a) Two-finger grasp of an ellipse along the contact normals. (b) Bounded segment $\mathcal{P}$ due to a vertical $\boldsymbol{f}_{\text {ext }}$. (c) $\mathcal{P}$ is a single point when $\boldsymbol{f}_{\text {ext }}$ is horizontal.

call that $x_{1}$ and $x_{2}$ denote the contact points, while $\left(t_{1}, n_{1}\right)$ and $\left(t_{2}, n_{2}\right)$ denote the tangent and normal directions at the contacts. Then, the kernel of $\bar{G}$ consists of all forces $\left(\boldsymbol{f}_{1}, \boldsymbol{f}_{2}\right)$ which act along the segment connecting $x_{1}$ and $x_{2}$ with equal magnitude and opposite directions. In this example, the line segment connecting $x_{1}$ and $x_{2}$ forms a $45^{\circ}$ angle with $t_{i}$ and $n_{i}(i=1,2)$. Hence, the tangent and normal components of the forces in the kernel of $\bar{G}$ are $\left(\boldsymbol{f}_{1} \cdot t_{1}, \boldsymbol{f}_{1} \cdot n_{1}, \boldsymbol{f}_{2} \cdot t_{2}, \boldsymbol{f}_{2}\right.$. $\left.n_{2}\right)=\nu(1,1,-1,1)$, where $\nu$ is an arbitrary scalar. Setting $\boldsymbol{w}_{\text {ext }}=\left(f_{\text {ext }}, 0\right)$, the solution $-\bar{G}^{\dagger} \boldsymbol{w}_{\text {ext }}$ is given by $-\bar{G}^{\dagger} \boldsymbol{w}_{\text {ext }}=$ $\left(f_{\text {ext }} / 2 \sqrt{2}\right)(-1,1,1,1)$, where $f_{\text {ext }}$ denotes the magnitude of $f_{\text {ext }}$. As depicted in Fig. 5(a), this solution consists of two horizontal forces of magnitude $(1 / 2) f_{\text {ext }}$ which together oppose the external force.

Using the expressions for the kernel of $\bar{G}$ and $-\bar{G}^{\dagger} \boldsymbol{w}_{\text {ext }}$, the bounded segment $\mathcal{P}=U \cap V$ corresponds to all solutions $\nu$ of the equation

$$
\begin{aligned}
&\left(\begin{array}{c}
1 \\
1 \\
-1 \\
1
\end{array}\right) \nu+\frac{f_{\text {ext }}}{2 \sqrt{2}}\left(\begin{array}{c}
-1 \\
1 \\
1 \\
1
\end{array}\right)=-\left[\begin{array}{ccc}
\sigma_{1} & & \\
& \ddots & \\
& & \sigma_{4}
\end{array}\right] \bar{G}^{T} \dot{q} \\
& \text { where } \dot{q} \in \mathbb{R}^{3} \text { and } \sigma_{1}, \ldots, \sigma_{4}>0 .
\end{aligned}
$$

According to Lemma 3.4, the endpoints of $\mathcal{P}$ lie on the coordinate hyperplanes in $\Delta f$-space. Equating the four scalar equations on the left-hand side (LHS) of (14) with zero, we obtain two pairs of identical equations: $\nu-\left(f_{\text {ext }} / 2 \sqrt{2}\right)=0$ and $\nu+\left(f_{\text {ext }} / 2 \sqrt{2}\right)=0$. Solving these equations for $\nu$, we obtain the following description of $\mathcal{P}$ :

$\mathcal{P}=\left\{\left(\begin{array}{c}\Delta f_{1}^{t} \\ \Delta f_{1}^{n} \\ \Delta f_{2}^{t} \\ \Delta f_{2}^{n}\end{array}\right)=\left(\begin{array}{c}1 \\ 1 \\ -1 \\ 1\end{array}\right) \nu+\frac{f_{\text {ext }}}{2 \sqrt{2}}\left(\begin{array}{c}-1 \\ 1 \\ 1 \\ 1\end{array}\right):-\frac{f_{\text {ext }}}{2 \sqrt{2}} \leq \nu \leq \frac{f_{\text {ext }}}{2 \sqrt{2}}\right\}$.

The force changes corresponding to $\mathcal{P}$ are depicted in Fig. 5(b). The force changes at $x_{1}$ are given by

$$
\begin{array}{r}
\left(\begin{array}{c}
\Delta f_{1}^{t} \\
\Delta f_{1}^{n}
\end{array}\right)=\left(\begin{array}{l}
1 \\
1
\end{array}\right) \nu+\left(f_{\text {ext }} / 2 \sqrt{2}\right)\left(\begin{array}{c}
-1 \\
1
\end{array}\right) \\
\text { such that }-\left(f_{\text {ext }} / 2 \sqrt{2}\right) \leq \nu \leq\left(f_{\text {ext }} / 2 \sqrt{2}\right) .
\end{array}
$$

These forces span the sector bounded by $-t_{1}$ and $n_{1}$. The force changes at $x_{2}$ are given by

$$
\left(\begin{array}{c}
\Delta f_{2}^{t} \\
\Delta f_{2}^{n}
\end{array}\right)=\left(\begin{array}{c}
-1 \\
1
\end{array}\right) \nu+\left(f_{\text {ext }} / 2 \sqrt{2}\right)\left(\begin{array}{l}
1 \\
1
\end{array}\right)
$$

with $\nu$ varying in the same interval. These forces span the sector bounded by $t_{2}$ and $n_{2}$. Note that the normal components $\Delta f_{1}^{n}$ and $\Delta f_{2}^{n}$ are nonnegative for all $-\left(f_{\text {ext }} / 2 \sqrt{2}\right) \leq \nu \leq$ $\left(f_{\text {ext }} / 2 \sqrt{2}\right)$. Hence, for any preloading of the grasp, none of the contacts is broken in response to $\boldsymbol{f}_{\text {ext }}$. The possibility that a contact would break due to insufficient initial preloading is discussed in Section V-A.

Example 2: The second example, shown in Fig. 6(a), is an ellipse held by two disc fingers along the contact normals $n_{1}$ and $n_{2}$. This particular equilibrium grasp can be established without any friction at the contacts, and is often preferred by automatic grasping systems. The kernel of $\bar{G}$ is 1-D, as before. However, in this grasp, the forces in the kernel of $\bar{G}$ are aligned with the contact normals. The kernel is thus spanned by $\nu(0,1,0,1)$, where $\nu$ is an arbitrary scalar. We consider two orientations of an external force acting on $\mathcal{B}$.

First, consider a vertical $f_{\text {ext }}$ which acts downward at the ellipse's center. Setting $\boldsymbol{w}_{\text {ext }}=\left(\boldsymbol{f}_{\text {ext }}, 0\right)$, the solution $-\bar{G}^{\dagger} \boldsymbol{w}_{\text {ext }}$ consists of pure normal forces and is given by $-\bar{G}^{\dagger} \boldsymbol{w}_{\text {ext }}=$ $\left(f_{\text {ext }} / 2\right)(0,1,0,-1)$. Since the kernel of $\bar{G}$ also consists of pure normal forces, we can depict the entire affine subspace $U$ in the plane of normal force components. This plane has coordinates $\left(\Delta f_{1}^{n}, \Delta f_{2}^{n}\right)$ and is obtained by intersecting $\left(\Delta f_{1}, \Delta f_{2}\right)$-space with the hyperplanes $\Delta f_{1}^{t}=0$ and $\Delta f_{2}^{t}=0$. Let $( \pm 1, \pm 1)$ denote the four quadrants in this plane. As shown in Fig. 6(b), the intersection of the feasible set $V$ with the hyperplanes $\Delta f_{1}^{t}=0$ and $\Delta f_{2}^{t}=0$ consists of the quadrants $(-1,1)$ and $(1,-1)$. The affine subspace $U$ intersects only the quadrant $(1,-1)$. Hence, the endpoints of $\mathcal{P}=U \cap V$ are located at $\left(\Delta f_{1}^{n}, \Delta f_{2}^{n}\right)=$ $\left(0,-f_{\text {ext }}\right)$ and $\left(\Delta f_{1}^{n}, \Delta f_{2}^{n}\right)=\left(f_{\text {ext }}, 0\right)$. The reaction contact forces induced by $f_{\text {ext }}$ are thus pure normal forces, given by $\boldsymbol{f}_{1}=\left(f_{1}^{n}\left(q_{0}\right)+\nu\right) n_{1}$ and $\boldsymbol{f}_{2}=\left(f_{2}^{n}\left(q_{0}\right)-\nu\right) n_{2}$ such that $\nu \in$ $\left[0, f_{\text {ext }}\right]$. Note that the initial preloading must satisfy $f_{i}^{n}\left(q_{0}\right) \geq$ $f_{\text {ext }}(i=1,2)$ so that the contact at $x_{2}$ would not break in response to $f_{\text {ext }}$ (see Section V-A).

Next, consider the application of a horizontal force $f_{\text {ext }}$ at the ellipse's center. In this case, the bounded segment $\mathcal{P}$ shrinks to a single point as follows. The solution $-\bar{G}^{\dagger} \boldsymbol{w}_{\text {ext }}$ consists of pure tangential forces and is given by $-\bar{G}^{\dagger} w_{\text {ext }}=\left(f_{\text {ext }} / 2\right)(-1,0,1,0)$. It follows that the affine subspace $U$ lies in the $\left(\Delta f_{1}^{n}, \Delta f_{2}^{n}\right)$-plane which is embedded at $\Delta f_{1}^{t}=-(1 / 2) f_{\text {ext }}$ and $\Delta f_{2}^{t}=(1 / 2) f_{\text {ext }}$ in $\left(\Delta f_{1}, \Delta f_{2}\right)$-space. The solution $-\bar{G}^{\dagger} \boldsymbol{w}_{\text {ext }}$ coincides with the origin in this plane, while the feasible set $V$ fills the quadrants $(-1,1)$ and $(1,-1)$. As depicted in Fig. 6(c), this is a nongeneric case where the affine subspace $U$ intersects only the boundary of the feasible set $V$. As a result, the bounded segment $\mathcal{P}$ shrinks to a single point which coincides with $-\bar{G}^{\dagger} \boldsymbol{w}_{\text {ext }}$. 


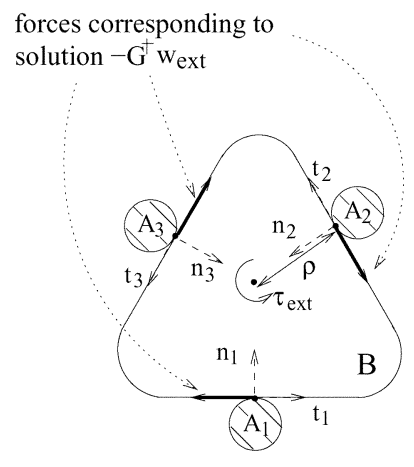

(a)

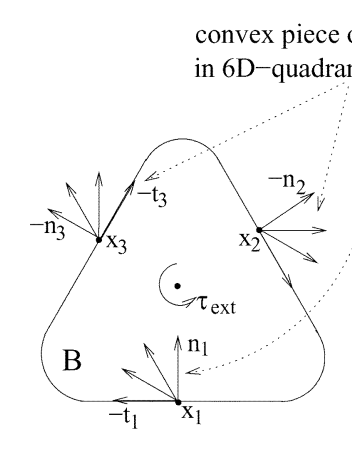

(b)

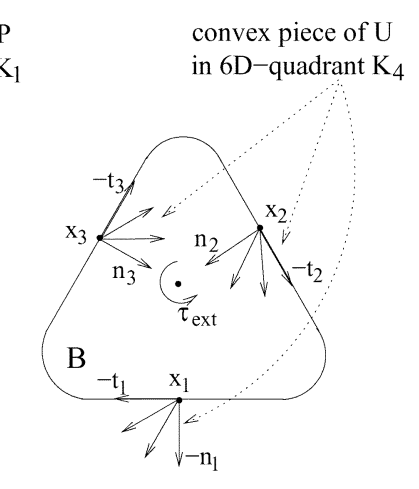

Fig. 7. (a) Three-finger grasp of a triangular object. (b) Forces corresponding to the portions of $\mathcal{P}$ in the $6 D$-quadrants $K_{1}$ and $K_{4}$.

The reaction forces due to a horizontal $f_{\text {ext }}$ are therefore unique, and are given by $\boldsymbol{f}_{1}=f_{1}^{n}\left(q_{0}\right) n_{1}-(1 / 2) f_{\text {ext }} t_{1}$ and $\boldsymbol{f}_{2}=f_{2}^{n}\left(q_{0}\right) n_{2}+(1 / 2) f_{\text {ext }} t_{2}$. However, any perturbation in the direction of $\boldsymbol{f}_{\text {ext }}$ yields a bounded segment of indeterminate reaction forces rather than a unique solution. Finally, the selection of preload forces that would prevent contact slippage in response to $\boldsymbol{f}_{\text {ext }}$ is discussed in Section V-B.

Example 3: The third example, depicted in Fig. 7(a), is a triangular object which is symmetrically preloaded by three disc fingers along the contact normals. The object is subjected to an external torque $\tau_{\text {ext }}$ that acts about the center. There are three frictional contacts in this example, hence contact force space is 6-D. The grasp matrix $\bar{G}$ is $3 \times 6$, and its 3 -D kernel is spanned by the following basis vectors. Every pair of contacts contributes one basis vector, which is given by two forces that act in opposite directions along the line segment connecting the two contacts. The tangent and normal components of the three basis vectors comprise the columns of the $6 \times 3$ matrix $E$, which is written below. Next, consider the solution $-\bar{G}^{\dagger} \boldsymbol{w}_{\text {ext }}$, where $\boldsymbol{w}_{\text {ext }}=\left(0, \tau_{\text {ext }}\right)$. Let $\rho$ denote the distance from the object's center to the three contacts. Then, $-\bar{G}^{\dagger} \boldsymbol{w}_{\text {ext }}$ is given by three tangent forces of equal magnitude, which together oppose the external torque [Fig. 7(a)]. The expressions for $E$ and $-\bar{G}^{\dagger} \boldsymbol{w}_{\text {ext }}$ are written in the following equation, that represents the intersection $U$ with $V$ :

$$
\begin{aligned}
\left(\begin{array}{ccc}
0 & -1 & 1 \\
0 & \sqrt{3} & \sqrt{3} \\
1 & 0 & -1 \\
\sqrt{3} & 0 & \sqrt{3} \\
-1 & 1 & 0 \\
\sqrt{3} & \sqrt{3} & 0
\end{array}\right)\left(\begin{array}{l}
\nu_{1} \\
\nu_{2} \\
\nu_{3}
\end{array}\right)-\frac{\tau_{\text {ext }}}{3 \rho}\left(\begin{array}{l}
1 \\
0 \\
1 \\
0 \\
1 \\
0
\end{array}\right) \\
=-\left[\begin{array}{lll}
\sigma_{1} & & \\
& \ddots & \\
& & \sigma_{6}
\end{array}\right] \bar{G}^{T} \dot{q}
\end{aligned}
$$

where $\dot{q} \in \mathbb{R}^{3}$ and $\sigma_{1}, \ldots, \sigma_{6}>0$. The feasible set $V$ fills entire 6-D quadrants in $\Delta f$-space (Lemma B.1). In order to determine the set $\mathcal{P}$, we have to determine which 6-D quadrants occupied by $V$ are intersected by $U$. Let $( \pm 1, \ldots, \pm 1)$ denote the $2^{6}=64$ possible 6-D quadrants in $\Delta f$-space. An analysis whose details are omitted here reveals that $V$ occupies 24 of the 6-D quadrants, while $U$ intersects six of these
6-D quadrants. The six 6-D quadrants, denoted $K_{1}, \ldots, K_{6}$, consist of two triplets: $K_{1}=(-1,1,-1,-1,-1,-1), K_{2}=$ $(-1,-1,-1,1,-1,-1), K_{3}=(-1,-1,-1,-1,-1,1)$, and $K_{4}=(-1,-1,-1,1,-1,1), K_{5}=(-1,1,-1,-1,-1,1)$, $K_{6}=(-1,1,-1,1,-1,-1)$. Note that the signs in each triplet are cyclically symmetric, reflecting the cyclic symmetry of the grasp arrangement. The forces corresponding to $K_{1}$ and $K_{4}$ are depicted in Fig. 7(b). The forces corresponding to $K_{2}$ and $K_{3}$ are cyclic permutations of the ones depicted for $K_{1}$, while the forces of $K_{5}$ and $K_{6}$ are cyclic permutations of the ones depicted for $K_{4}$.

We now construct the linear inequalities that define the convex pieces of $\mathcal{P}$. Each convex piece of $\mathcal{P}$ is associated with a particular $6 D$-quadrant $K_{i}$. According to Lemma 3.4, the planar facets of each piece are embedded in the coordinate hyperplanes of $\Delta f$-space. The representation of these facets in the null space of $\bar{G}$ is obtained by equating the six equations on the LHS of (15) to zero. Next we convert the six equalities into inequalities according to the sign vector of the particular 6-D quadrant under consideration. For instance, the piece of $\mathcal{P}$ in $K_{1}=(-1,1,-1,-1,-1,-1)$ is parametrized by the inequalities

$$
\mathcal{P} \cap K_{1}=\left\{\left(\begin{array}{c}
\nu_{1} \\
\nu_{2} \\
\nu_{3}
\end{array}\right) \in \mathbb{R}^{3}: \begin{array}{cc}
-\nu_{2}+\nu_{3}-\frac{\tau_{\mathrm{ext}}}{3 \rho} & \leq 0 \\
\nu_{2}+\nu_{3} & \geq 0 \\
\nu_{1}-\nu_{3}-\frac{\tau_{\mathrm{ext}}}{3 \rho} & \leq 0 \\
\nu_{1}+\nu_{3} & \leq 0 \\
-\nu_{1}+\nu_{2}-\frac{\tau_{\mathrm{ext}}}{3 \rho} & \leq 0 \\
\nu_{1}+\nu_{2} & \leq 0
\end{array}\right\} .
$$

The set $\mathcal{P}$ resulting from the union of convex polyhedra $\cup_{i=1}^{6} \mathcal{P} \cap K_{i}$ is depicted in Figs. 8 and 9 . Note that both figures use the null-space parametrization of $\bar{G}$ by $\nu \in \mathbb{R}^{3}$. The set $\mathcal{P}$ is obtained by mapping the sets depicted in the figures into $\Delta f$-space. Finally, here too the preload forces must be sufficiently high to ensure that none of the contacts would break or slip in response to $\tau_{\text {ext }}$. This topic is discussed in the next section.

\section{USING THE POLYHEDRAL BOUND FOR GRASP SYNTHESIS}

In this section, we discuss the selection of preload forces such that none of the contacts breaks or slips in response to an applied external wrench. The discussion is divided into three parts. First, we discuss the prevention of contact breakage in response to 


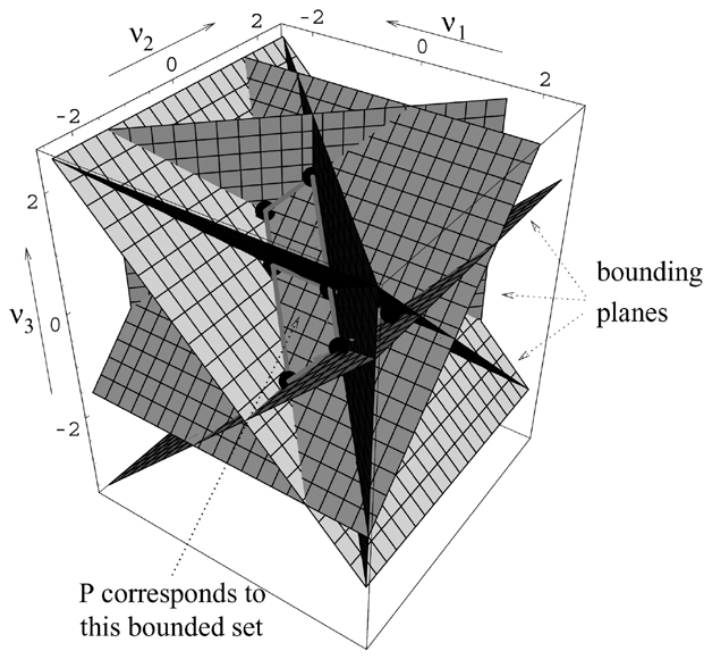

Fig. 8. Null-space parametrization of $\mathcal{P}$ for the three-finger grasp.
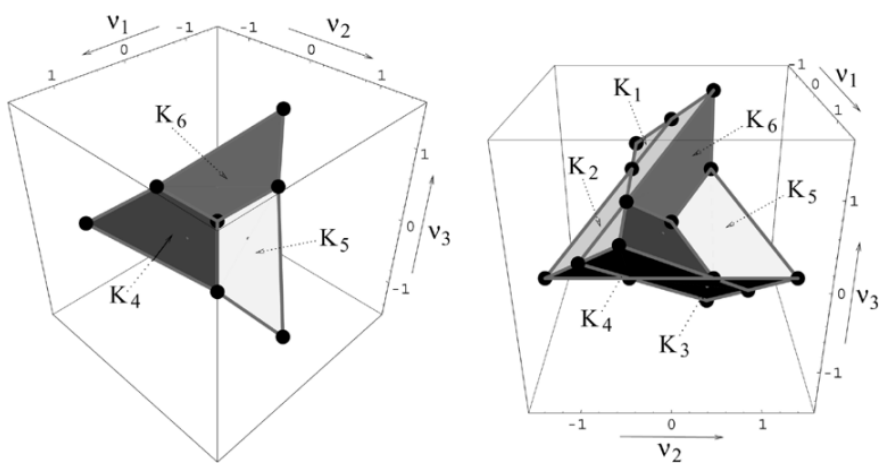

Fig. 9. Two top views of the null-space parametrization of $\mathcal{P}$ for the three-finger grasp.

$\boldsymbol{w}_{\text {ext }}$. Next, we discuss the prevention of contact slippage in response to $\boldsymbol{w}_{\text {ext }}$. Finally, we introduce the notion of robust grasp synthesis, which is concerned with selection of preload forces such that a given set of wrenches is passively resisted by the contacts. The selection of preload forces is based on the following formula. When an external wrench $\boldsymbol{w}_{\text {ext }}$ acts on an object held in a preloaded grasp, the contact reaction forces induced by $w_{\text {ext }}$ are given by

$$
\left(\begin{array}{l}
f^{t} \\
f^{n}
\end{array}\right)=\left(\begin{array}{c}
f^{t}\left(q_{0}\right) \\
f^{n}\left(q_{0}\right)
\end{array}\right)+\left(\begin{array}{c}
\Delta f^{t} \\
\Delta f^{n}
\end{array}\right) \quad\left(\Delta f^{t}, \Delta f^{n}\right) \in \mathcal{P}
$$

where $\mathcal{P}$ is the bounded polyhedral set. The formula specifies a bounded polyhedral set with a basepoint at the preload forces $f\left(q_{0}\right)=\left(f^{t}\left(q_{0}\right), f^{n}\left(q_{0}\right)\right)$. In the following, the set specified by (16) is denoted $f\left(q_{0}\right)+\mathcal{P}$.

\section{A. Positive Normal Load Constraint}

First, consider the selection of preload forces such that none of the contacts would break in response to $\boldsymbol{w}_{\text {ext }}$. Let $H$ be the set of contact forces having a nonnegative normal component

$$
H=\left\{\left(\begin{array}{c}
f^{t} \\
f^{n}
\end{array}\right)=\left(\begin{array}{c}
f^{t}\left(q_{0}\right) \\
f^{n}\left(q_{0}\right)
\end{array}\right)+\left(\begin{array}{c}
\Delta f^{t} \\
\Delta f^{n}
\end{array}\right): f^{n}\left(q_{0}\right)+\Delta f^{n} \geq \overrightarrow{0}\right\}
$$

where $v \geq \overrightarrow{0}$ means that each component of the vector $v$ is nonnegative. If $f\left(q_{0}\right)+\mathcal{P}$ is contained in $H$ [see Fig. 10(a)], none of

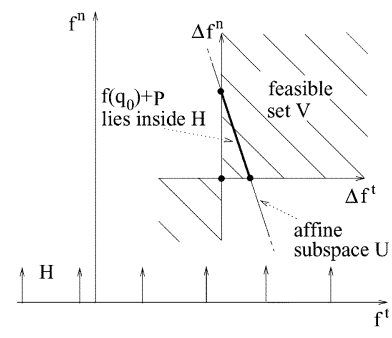

(a)

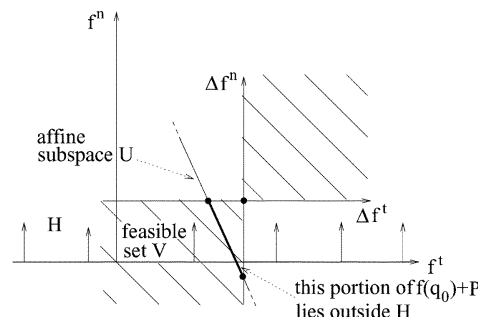

(b)
Fig. 10. Two intersection arrangements of $f\left(q_{0}\right)+\mathcal{P}$ with $H$, corresponding to different normal preloadings.

the contacts would break in response to $\boldsymbol{w}_{\text {ext }}$. If $f\left(q_{0}\right)+\mathcal{P}$ is only partially contained in $H$ [see Fig. 10(b)], some of the reaction forces can assume negative normal loading in response to $\boldsymbol{w}_{\text {ext }}$. The corresponding contacts can break, and the response to $\boldsymbol{w}_{\text {ext }}$ would become unpredictable. For purposes of grasp synthesis, we assert the following fact. For any given $\boldsymbol{w}_{\text {ext }}$, there exist sufficiently high preload forces such that $f\left(q_{0}\right)+\mathcal{P}$ is completely contained in $H$. The selection of suitable preload forces is illustrated in the following example.

Example 4: Consider the two-finger grasp of an ellipse discussed in Example 1. Let the ellipse be subjected to a force $f_{\text {ext }}$ which acts downward at the ellipse's center [see Fig. 11(a)]. The kernel of $\bar{G}$ is the same as in Example 1. The solution $-\bar{G}^{\dagger} \boldsymbol{w}_{\text {ext }}$ requires two parameters depicted in Fig. 11(a). Let the object-frame origin be located at the intersection point of the normals $n_{1}$ and $n_{2}$. The first parameter $\rho_{0}$ is the distance from the object's origin to the ellipse's center. The second parameter $\rho_{1}$ is the distance from the object's origin to the contacts $x_{1}$ and $x_{2}$. Using these parameters, it can be verified that

$-\bar{G}^{\dagger} \boldsymbol{w}_{\mathrm{ext}}=\frac{1}{2} \frac{\rho_{0}}{\rho_{1}} f_{\mathrm{ext}}\left(\begin{array}{c}-1 \\ 0 \\ -1 \\ 0\end{array}\right)+\left(\frac{1}{\sqrt{2}}+\frac{1}{2} \frac{\rho_{0}}{\rho_{1}}\right) f_{\mathrm{ext}}\left(\begin{array}{c}0 \\ 1 \\ 0 \\ -1\end{array}\right)$.

(The first summand opposes the torque generated by $\boldsymbol{f}_{\text {ext }}$ about the object's origin, while the second summand opposes the forces generated by the first summand and $f_{\text {ext }}$.) Substituting the expressions for the kernel of $\bar{G}$ and $-\bar{G}^{\dagger} w_{\text {ext }}$, the bounded segment $\mathcal{P}=U \cap V$ corresponds to all solutions $\nu$ of the equation

$$
\begin{array}{r}
\left(\begin{array}{c}
1 \\
1 \\
-1 \\
1
\end{array}\right) \nu+\frac{1}{2} \frac{\rho_{0}}{\rho_{1}} f_{\text {ext }}\left(\begin{array}{c}
-1 \\
0 \\
-1 \\
0
\end{array}\right)+\left(\frac{1}{\sqrt{2}}+\frac{1}{2} \frac{\rho_{0}}{\rho_{1}}\right) f_{\text {ext }}\left(\begin{array}{c}
0 \\
1 \\
0 \\
-1
\end{array}\right) \\
=-\left[\begin{array}{ccc}
\sigma_{1} & \\
& \ddots & \\
& & \sigma_{4}
\end{array}\right] \bar{G}^{T} \dot{q} \quad \dot{q} \in \mathbb{R}^{3}, \sigma_{1}, \ldots, \sigma_{4}>0 .
\end{array}
$$

Equating the LHS of the resulting equation to zero gives four scalar equations in $\nu$. The solutions of the four equations are $\nu=$ $\pm(1 / 2)\left(\rho_{0} / \rho_{1}\right) f_{\text {ext }}$ and $\nu= \pm\left((1 / \sqrt{2})+(1 / 2)\left(\rho_{0} / \rho_{1}\right)\right) f_{\text {ext }}$, where $f_{\text {ext }}$ is the magnitude of $f_{\text {ext }}$. These solutions parametrize the candidate endpoints of the bounded segment $\mathcal{P}$. According to Lemma 3.3, $\mathcal{P}$ is connected and contains the solution 


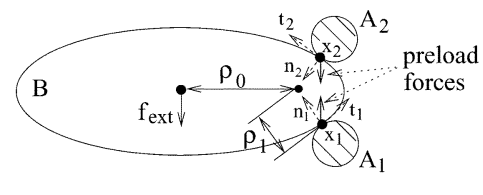

(a)

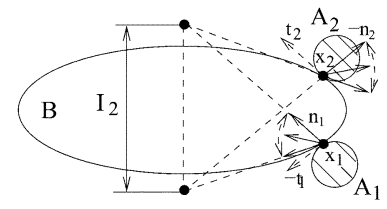

(b)

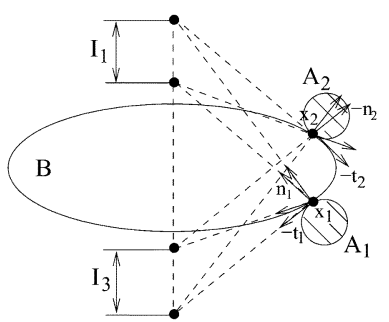

Fig. 11. (a) Ellipse grasped by two fingers is subjected to a downward force $f_{\text {ext }}$. (b) Contact force changes in $\mathcal{P}$ are parametrized by $\nu \in I_{1} \cup I_{2} \cup I_{3}$.

$-\bar{G}^{\dagger} \boldsymbol{w}_{\text {ext. }}$. Since $-\bar{G}^{\dagger} \boldsymbol{w}_{\text {ext }}$ corresponds to $\nu=0$, the range of $\nu$ that parametrizes $\mathcal{P}$ must include the innermost interval $I_{2}=$ $\left[-(1 / 2)\left(\rho_{0} / \rho_{1}\right) f_{\text {ext }},(1 / 2)\left(\rho_{0} / \rho_{1}\right) f_{\text {ext }}\right]$. The outer intervals, $I_{1}=\left[-\left((1 / \sqrt{2})+(1 / 2)\left(\rho_{0} / \rho_{1}\right)\right) f_{\text {ext }},-(1 / 2)\left(\rho_{0} / \rho_{1}\right) f_{\text {ext }}\right]$ and $I_{3}=\left[(1 / 2)\left(\rho_{0} / \rho_{1}\right) f_{\text {ext }},\left((1 / \sqrt{2})+(1 / 2)\left(\rho_{0} / \rho_{1}\right)\right) f_{\text {ext }}\right]$, parametrize segments that lie in specific 4-D quadrants in $\Delta f$-space. Let $( \pm 1, \pm 1, \pm 1, \pm 1)$ denote the 16 possible 4 -D quadrants in this space. Then the segments parametrized by $I_{1}$ and $I_{3}$ lie in the 4-D quadrants $(-1,1,1,-1)$ and $(1,1,-1,-1)$. Moreover, both of these 4-D quadrants are occupied by the feasible set. It follows that $\mathcal{P}$ is parametrized by the interval $I=I_{1} \cup I_{2} \cup I_{3}$. Summarizing this example, the set $\mathcal{P}$ due to a vertical $f_{\text {ext }}$ is

$$
\begin{array}{r}
\mathcal{P}=\left\{\left(\begin{array}{c}
\Delta f_{1}^{t} \\
\Delta f_{1}^{n} \\
\Delta f_{2}^{t} \\
\Delta f_{2}^{n}
\end{array}\right)=\left(\begin{array}{c}
1 \\
1 \\
-1 \\
1
\end{array}\right) \nu+\frac{1}{2} \frac{\rho_{0}}{\rho_{1}} f_{\operatorname{ext}}\left(\begin{array}{c}
-1 \\
0 \\
-1 \\
0
\end{array}\right)\right. \\
\left.+\left(\frac{1}{\sqrt{2}}+\frac{1}{2} \frac{\rho_{0}}{\rho_{1}}\right) f_{\operatorname{ext}}\left(\begin{array}{c}
0 \\
1 \\
0 \\
-1
\end{array}\right): \nu \in I\right\}
\end{array}
$$

such that $I=\left[-\left((1 / \sqrt{2})+(1 / 2)\left(\rho_{0} / \rho_{1}\right)\right) f_{\text {ext }},((1 / \sqrt{2})+\right.$ $\left.\left.(1 / 2)\left(\rho_{0} / \rho_{1}\right)\right) f_{\text {ext }}\right]$. The forces corresponding to $\mathcal{P}$ are depicted in Fig. 11(b). It is intuitively clear that the action of $f_{\text {ext }}$ on $\mathcal{B}$ reduces the normal loading at $x_{2}$. Indeed, (17) tells us that $\Delta f_{2}^{n}=\nu-\left((1 / \sqrt{2})+(1 / 2)\left(\rho_{0} / \rho_{1}\right)\right) f_{\text {ext }} \leq 0$ when $\nu$ varies in $I$. In order to prevent breakage of the contact at $x_{2}$ in response to $\boldsymbol{f}_{\text {ext }}$, the normal preloading at both contacts must satisfy $f_{i}^{n}\left(q_{0}\right) \geq\left((1 / \sqrt{2})+(1 / 2)\left(\rho_{0} / \rho_{1}\right)\right) f_{\text {ext }}$ for $i=1,2$.

\section{B. Friction-Cone Constraint}

Next, consider the selection of preload forces that would prevent the contacts from slipping in response to $\boldsymbol{w}_{\text {ext }}$. Let $\mathrm{FC}_{i}$ be the set of contact reaction forces at $x_{i}$ satisfying the $i$ th friction-cone constraint

$$
\begin{array}{r}
\mathrm{FC}_{i}=\left\{\left(\begin{array}{c}
f_{i}^{t}\left(q_{0}\right) \\
f_{i}^{n}\left(q_{0}\right)
\end{array}\right)+\left(\begin{array}{c}
\Delta f_{i}^{t} \\
\Delta f_{i}^{n}
\end{array}\right): f_{i}^{n}\left(q_{0}\right)+\Delta f_{i}^{n}>0\right. \\
\text { and } \left.\left|f_{i}^{t}\left(q_{0}\right)+\Delta f_{i}^{t}\right| \leq \mu\left(f_{i}^{n}\left(q_{0}\right)+\Delta f_{i}^{n}\right)\right\}
\end{array}
$$

where $\mu$ is the coefficient of friction. Let $\mathrm{FC}=\mathrm{FC}_{1} \times \cdots \times \mathrm{FC}_{k}$ be the set of contact forces that simultaneously satisfy the friction-cone constraints at the $k$ contacts. Note that, by definition, FC is contained in the positive-normal-load set $H$. If $f\left(q_{0}\right)+\mathcal{P}$ is not completely contained in FC, some of the contacts may slip, and the response to $\boldsymbol{w}_{\text {ext }}$ would be unpredictable. For the purpose of grasp synthesis, the following lemma asserts that it is always possible to select preload forces such that $f\left(q_{0}\right)+\mathcal{P}$ is contained in FC. The lemma is based on the notion of force closure. A $k$-finger grasp is force closure if the contact forces can generate any net wrench on the grasped object [26].

Lemma 5.1: Let $\mathcal{B}$ be held in an equilibrium grasp by $k$ fingers. If the grasp is force closure, for any $w_{\text {ext }}$, there exist sufficiently high preload forces such that $f\left(q_{0}\right)+\mathcal{P}$ is completely contained in FC.

Proof: In general, a planar grasp is force closure iff the grasping forces lie in the interior of the respective friction cones [20], [43]. Hence, at each contact $x_{i}$, there exists a scalar $0 \leq s_{i}<1$, such that $\left|f_{i}^{t}\left(q_{0}\right)\right|=s_{i} \mu f_{i}^{n}\left(q_{0}\right)$. Next, we define two bounds on the force changes at $x_{i}$. The first is a lower bound on the normal component $m_{i}=\min \left\{\Delta f_{i}^{n}\right\}$ over all $\left(\Delta f_{i}^{t}, \Delta f_{i}^{n}\right) \in \mathcal{P}$. The second is an upper bound on the absolute value of the tangent component $M_{i}=\max \left\{\left|\Delta f_{i}^{t}\right|\right\}$ over all $\left(\Delta f_{i}^{t}, \Delta f_{i}^{n}\right) \in \mathcal{P}$. Both $m_{i}$ and $M_{i}$ are finite numbers since $\mathcal{P}$ is a bounded set in $\Delta f$-space. Next, we split the normal preload $f_{i}^{n}\left(q_{0}\right)$ into three summands: $f_{i}^{n}\left(q_{0}\right)=$ $(1 / 2)\left(1-s_{i}\right) f_{i}^{n}\left(q_{0}\right)+(1 / 2)\left(1-s_{i}\right) f_{i}^{n}\left(q_{0}\right)+s_{i} f_{i}^{n}\left(q_{0}\right)$. We use the first summand to ensure that the net normal force at $x_{i}$ is positive: $(1 / 2)\left(1-s_{i}\right) f_{i}^{n}\left(q_{0}\right)+m_{i}>0$. We use the remaining two summands to ensure that the net tangent force at $x_{i}$ satisfies the friction-cone constraint: $\left|f_{i}^{t}\left(q_{0}\right)+\Delta f_{i}^{t}\right| \leq$ $\left|f_{i}^{t}\left(q_{0}\right)\right|+M_{i} \leq \mu\left(s_{i} f_{i}^{n}\left(q_{0}\right)+(1 / 2)\left(1-s_{i}\right) f_{i}^{n}\left(q_{0}\right)\right)$. Since $\left|f_{i}^{t}\left(q_{0}\right)\right|=s_{i} \mu f_{i}^{n}\left(q_{0}\right)$, selecting the $i$ th normal preload such that $M_{i} \leq(1 / 2)\left(1-s_{i}\right) \mu f_{i}^{n}\left(q_{0}\right)$ ensures that the friction-cone constraint at $x_{i}$ is satisfied. The two inequalities on $f_{i}^{n}\left(q_{0}\right)$ can be written together in the formula

$$
f_{i}^{n}\left(q_{0}\right)>\frac{2}{\left(1-s_{i}\right) \mu} \max \left\{-m_{i}, M_{i}\right\}
$$

where $0 \leq s_{i}<1$. A selection of $f_{i}^{n}\left(q_{0}\right)$ according to this formula guarantees that the entire bounded set $f\left(q_{0}\right)+\mathcal{P}$ satisfies the $i$ th friction-cone constraint. Finally, the magnitude of the preload forces can be freely scaled, since by construction these forces lie in the kernel of $\bar{G}$.

The following example illustrates the computation of preload forces that would prevent contact slippage in response to $\boldsymbol{w}_{\text {ext }}$.

Example 5: Consider the two-finger grasp discussed in Example 1. Let a horizontal external force $\boldsymbol{f}_{\text {ext }}$ attempt to pull the ellipse away from the fingers. We wish to compute the preload forces that would prevent the contacts from slipping in response to $f_{\text {ext }}$. The kernel of $\bar{G}$ is the same as in Example 1. The solution $-\bar{G}^{\dagger} \boldsymbol{w}_{\text {ext }}$ consists of two horizontal forces of magnitude $(1 / 2) f_{\text {ext }}$ which oppose the external force, $-\bar{G}^{\dagger} \boldsymbol{w}_{\text {ext }}=$ 
$\left(f_{\text {ext }} / 2 \sqrt{2}\right)(1,-1,-1,-1)$. Using the steps described in $E x$ ample 1 , the bounded segment $\mathcal{P}$ is

$$
\begin{aligned}
& \mathcal{P}=\left\{\left(\begin{array}{c}
\Delta f_{1}^{t} \\
\Delta f_{1}^{n} \\
\Delta f_{2}^{t} \\
\Delta f_{2}^{n}
\end{array}\right)=\left(\begin{array}{c}
1 \\
1 \\
-1 \\
1
\end{array}\right) \nu+\frac{f_{\text {ext }}}{2 \sqrt{2}}\left(\begin{array}{c}
1 \\
-1 \\
-1 \\
-1
\end{array}\right)\right. \\
& \left.:-\frac{f_{\text {ext }}}{2 \sqrt{2}} \leq \nu \leq \frac{f_{\text {ext }}}{2 \sqrt{2}}\right\} \text {. }
\end{aligned}
$$

The computation now follows the steps described in the proof of Lemma 5.1. First, we determine the bounds $m_{i}$ and $M_{i}$ for $i=1,2$. Using (19), the normal force changes induced by $f_{\text {ext }}$ are $\Delta f_{1}^{n}=\Delta f_{2}^{n}=\nu-\left(f_{\text {ext }} / 2 \sqrt{2}\right) \geq-\left(f_{\text {ext }} / \sqrt{2}\right)$ in the interval $\nu \in\left[-\left(f_{\text {ext }} / 2 \sqrt{2}\right),\left(f_{\text {ext }} / 2 \sqrt{2}\right)\right]$. Hence, $m_{1}=m_{2}=-\left(f_{\text {ext }} / \sqrt{2}\right)$. The tangent force changes induced by $f_{\text {ext }}$ are $\Delta f_{1}^{t}=\nu+\left(f_{\text {ext }} / 2 \sqrt{2}\right)$ and $\Delta f_{2}^{t}=$ $-\nu-\left(f_{\text {ext }} / 2 \sqrt{2}\right)$. An upper bound on $\left|\Delta f_{1}^{t}\right|$ and $\left|\Delta f_{2}^{t}\right|$ in the interval $\nu \in\left[-\left(f_{\text {ext }} / 2 \sqrt{2}\right),\left(f_{\text {ext }} / 2 \sqrt{2}\right)\right]$ is thus $M_{1}=M_{2}=\left(f_{\text {ext }} / \sqrt{2}\right)$. Next, we determine the value of the scalars $s_{1}$ and $s_{2}$. The preload forces form $45^{\circ}$ with $n_{1}$ and $n_{2}$. Hence, $s_{1}=s_{2}=1 / \mu$, where $\mu$ is necessarily larger than unity to allow the preload forces to lie in the interior of the friction cones. Using the scalars $s_{i}$, the friction-cone constraints at $x_{i}$ are satisfied by the inequalities $(1 / 2)\left(1-s_{i}\right) \mu f_{i}^{n}\left(q_{0}\right)+m_{i}>0$ and $M_{i} \leq(1 / 2)\left(1-s_{i}\right) \mu f_{i}^{n}\left(q_{0}\right) \quad(i=1,2)$. Substituting $\left(1-s_{i}\right) \mu=\mu-1$, the normal component of the preload forces must satisfy $f_{i}^{n}\left(q_{0}\right) \geq \max \{1,1 /(\mu-1)\} \sqrt{2} f_{\text {ext }}$ for $i=1,2$, where $\mu>1$.

\section{Robust Grasp Synthesis}

Finally, consider the task of selecting preload forces such that the contacts will resist a set rather than a particular external wrench. This set can model an uncertainty neighborhood about a nominal wrench or it can model a time-varying wrench that acts on a fixtured workpiece during a particular machining stage. We describe how to compute the collection of indeterminate forces that can arise in response to a bounded convex polyhedral set of external wrenches. Let $\mathcal{W}$ denote the convex polyhedral set of wrenches, and let $\mathcal{P}_{w}$ denote the bounded polyhedral set of force changes induced by the action of a specific $\boldsymbol{w} \in \mathcal{W}$ on $\mathcal{B}$. We compute the union $\cup_{w \in \mathcal{W}} \mathcal{P}_{w}$ by considering its individual pieces in the $2 k$-quadrants of $\Delta f$-space. Recall that the feasible set $V$ fills entire $2 k$-quadrants in $\Delta f$-space (Lemma B.1). Moreover, the $2 k$-quadrants occupied by $V$ are fixed and do not depend on the specific external wrench acting on $\mathcal{B}$. Let $\mathcal{I}$ denote the index set of the $2 k$-quadrants occupied by $V$. Then, the collection of force changes induced by $\mathcal{W}$ is given by $\cup_{w \in \mathcal{W}} \mathcal{P}_{w}=\cup_{i \in \mathcal{I}} \cup_{w \in \mathcal{W}} \mathcal{P}_{w} \cap K_{i}$. For a given $\boldsymbol{w} \in \mathcal{W}$, each piece $\mathcal{P}_{w} \cap K_{i}$ is a convex polyhedral set. The following proposition asserts that this convexity is preserved when $\boldsymbol{w}$ varies in $\mathcal{W}$.

Proposition 3.2 ([34]): Let $\mathcal{B}$ be held by $k$ fingers in an equilibrium grasp. Let $K_{i}$ be a $2 k$-quadrant occupied by the feasible set $V$. If $\mathcal{W}$ is a bounded convex polyhedral set of wrenches, the collection of force changes $\cup_{w \in \mathcal{W}} \mathcal{P}_{w} \cap K_{i}$ is a bounded convex polyhedral set in $\Delta f$-space.

The proposition provides us with an efficient means for computing the collection of contact reaction forces induced by $\mathcal{W}$. First, for each $i \in \mathcal{I}$, one computes the convex piece $\cup_{w \in \mathcal{W}} \mathcal{P}_{w} \cap$

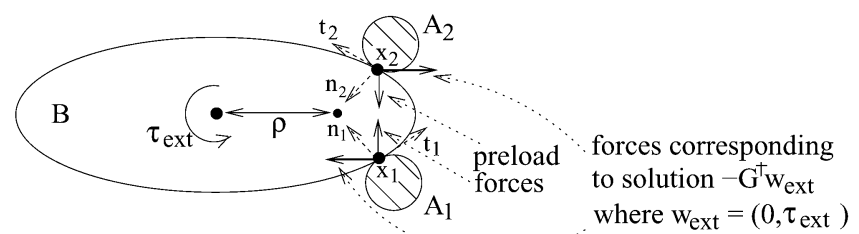

Fig. 12. Two-finger grasp of an ellipse which is subjected to a torque $\tau_{\text {ext }} \in$ $\left[\tau_{0}-\Delta \tau, \tau_{0}+\Delta \tau\right]$

$K_{i}$. Next, one takes the union $\cup_{w \in \mathcal{W}} \mathcal{P}_{w}=\cup_{i \in \mathcal{I}} \cup_{w \in \mathcal{W}} \mathcal{P}_{w} \cap K_{i}$. Finally, the contact reaction forces induced by $\mathcal{W}$ are obtained by adding the preload forces to the collection of force changes, $f=f\left(q_{0}\right)+\cup_{w \in \mathcal{W}} \mathcal{P}_{w}$. Once the set $f\left(q_{0}\right)+\mathcal{P}$ is known, one selects preload forces such that the entire set satisfies the friction-cone constraints discussed above. This last stage is illustrated with an example.

Example 6: Consider the two-finger grasp discussed in Example 1. The ellipse is subjected to a drilling torque $\tau_{\text {ext }}$ which acts at the ellipse's center in a counterclockwise direction (Fig. 12). Due to process uncertainties, $\tau_{\text {ext }}$ varies in an interval $\left[\tau_{0}-\Delta \tau, \tau_{0}+\Delta \tau\right]$, where $\tau_{0}$ a nominal torque and $\Delta \tau>0$ is a tolerance parameter. The corresponding set of external wrenches is given by $\mathcal{W}=\left\{\left(0, \tau_{\text {ext }}\right): \tau_{0}-\Delta \tau \leq \tau_{\text {ext }} \leq \tau_{0}+\Delta \tau\right\}$. This example is sufficiently simple so that we can directly solve for the collection of reaction forces induced by $\mathcal{W}$.

Since the kernel of $\bar{G}$ is $1-\mathrm{D}$, for each fixed $w=\left(0, \tau_{\text {ext }}\right)$, the set $\mathcal{P}_{w}$ is a bounded segment. The computation of $\mathcal{P}_{w}$ is similar to the one carried in Example 1. First, the kernel of $\bar{G}$ is spanned by $(1,1,-1,1)$. Second, for each $\boldsymbol{w} \in \mathcal{W}$, the solution $-\bar{G}^{\dagger} \boldsymbol{w}$ consists of two horizontal forces that act at $x_{1}$ and $x_{2}$ with equal magnitude and opposite directions (Fig. 12). The two forces generate a net torque $-\tau_{\text {ext }}$ on $\mathcal{B}$, and their components are given by $-\bar{G}^{\dagger} \boldsymbol{w}=\left(\tau_{\text {ext }} / 2 \sqrt{2} \rho\right)(-1,1,-1,-1)$, where $\rho$ is depicted in the figure. Substituting for $-\bar{G}^{\dagger} \boldsymbol{w}$ in (14) and then equating the LHS of the resulting equation to zero gives two identical pairs of equations in $\nu$. The solutions of these equations are $\nu= \pm\left(\tau_{\text {ext }} / 2 \sqrt{2} \rho\right)$, and $\mathcal{P}_{w}$ is given by

$\mathcal{P}_{w}=\left\{\left(\begin{array}{c}1 \\ 1 \\ -1 \\ 1\end{array}\right) \nu+\frac{\tau_{\text {ext }}}{2 \sqrt{2} \rho}\left(\begin{array}{c}-1 \\ 1 \\ -1 \\ -1\end{array}\right):-\frac{\tau_{\text {ext }}}{2 \sqrt{2} \rho} \leq \nu \leq \frac{\tau_{\text {ext }}}{2 \sqrt{2} \rho}\right\}$

Next, we parametrize the external torques by $\tau_{\text {ext }}(s)=\tau_{0}+$ $s \Delta \tau$ such that $-1 \leq s \leq 1$. The union of the segments $\mathcal{P}_{w}$ is given by

$$
\begin{aligned}
\bigcup_{w \in \mathcal{W}} \mathcal{P}_{w}= & \left\{\left(\begin{array}{c}
1 \\
1 \\
-1 \\
1
\end{array}\right) \nu+\frac{\tau_{\mathrm{ext}}(s)}{2 \sqrt{2} \rho}\left(\begin{array}{c}
-1 \\
1 \\
-1 \\
-1
\end{array}\right)\right. \\
& \left.:-\frac{\tau_{\mathrm{ext}}(s)}{2 \sqrt{2} \rho} \leq \nu \leq \frac{\tau_{\mathrm{ext}}(s)}{2 \sqrt{2} \rho} \text { and }-1 \leq s \leq 1\right\}
\end{aligned}
$$

In accordance with Proposition 5.2, $\cup_{w \in \mathcal{W}} \mathcal{P}_{w}$ is a 2-D convex polygon in $\Delta f$-space. We can now compute the 
preload forces that will prevent the contacts from breaking or slipping in response to any torque in $\mathcal{W}$. Using (21), $\Delta f_{1}^{n}=$ $\nu+\left(\tau_{\text {ext }}(s) / 2 \sqrt{2} \rho\right) \geq 0$ while $\Delta f_{2}^{n}=\nu-\left(\tau_{\text {ext }}(s)\right) /(2 \sqrt{2} \rho) \geq$ $-\left(\tau_{0}-\Delta \tau\right) /(\sqrt{2} \rho)$. Hence, the lower bounds on $\Delta f_{i}^{n}$ are $m_{1}=0$ and $m_{2}=-\left(\tau_{0}-\Delta \tau\right) /(\sqrt{2} \rho)$. Using (21) again, $\left|\Delta f_{1}^{t}\right|=\mid \nu-\left(\tau_{\text {ext }}(s)\right) /\left(2 \sqrt{2} \rho \mid \leq\left(\tau_{0}-\Delta \tau\right) /(\sqrt{2} \rho)\right.$ and $\left|\Delta f_{2}^{t}\right|=\left|-\nu-\left(\tau_{\text {ext }}(s)\right) /(2 \sqrt{2} \rho)\right| \leq\left(\tau_{0}-\Delta \tau\right) /(\sqrt{2} \rho)$. Hence, the upper bounds on $\left|\Delta f_{i}^{t}\right|$ are $M_{1}=M_{2}=\left(\tau_{0}-\Delta \tau\right) /(\sqrt{2} \rho)$. Substituting for $m_{i}$ and $M_{i}$ in (18), the normal component of the preload forces must satisfy $f_{i}^{n} \geq \max \left\{(1 / 2) M_{i},\left(1-s_{i}\right) m_{i}\right\}$ for $i=1,2$. These preload forces guarantee that the two contacts will passively resist any torque in $\mathcal{W}$.

Finally, let us put the grasp synthesis method in the context of the existing literature. The synthesis method is most relevant for fixturing and industrial gripping applications, where sophisticated contact-force sensors and agile controllers are not necessarily available. The literature relevant for such applications focuses on synthesizing grasps that merely satisfy a specific stability criterion, such as form-closure (e.g., [8]) or potential-energy minimization (e.g., [38]) in frictionless grasps, and force-closure in frictional grasps (e.g., [21] and [25]). However, without actively measuring and controlling the contact forces, the resulting grasps provide passive stabilization for some unspecified and perhaps small neighborhood of disturbance wrenches. Furthermore, when a grasp is statically indeterminate, it is not clear how to select the preload forces magnitudes. In contrast, the synthesis method discussed here specifies how to select suitable preload forces which guarantee that all disturbance wrenches in an allowed convex set would be passively rejected by the contacts.

\section{CONCLUSION}

When an external wrench acts on a perfectly rigid object grasped by perfectly rigid finger bodies, the statically feasible contact reaction forces typically span an unbounded linear space. However, in reality all bodies exhibit some degree of compliance due to material deformation. Under a quasi-rigid body framework these deformations occur only in the vicinity of the contacts. We described two generic rules that govern the force-displacement relationship at a quasi-rigid contact. These rules govern the behavior of a wide range of compliance models and make no prior assumptions on the specific value of the contact parameters. When the effect of these rules at the contacts is coupled through the rigid-body kinematics of the grasped object, the statically feasible reaction forces span a bounded polyhedral set. Since the polyhedral bound is obtained without specific knowledge on the contact parameters, it is useful for robust grasp and fixture synthesis in highly variable environments. Given a bounded set of external wrenches that can act on the grasped object, one first computes the bounded set of reaction forces induced by this set. In particular, when the external wrenches form a convex neighborhood, the reaction forces can be efficiently computed as a convex combination of the polyhedral sets associated with the vertices of the convex neighborhood. Moreover, we showed that one can always select preload forces such that the entire collection of reaction forces satisfies the friction-cone constraints at the contacts. The resulting grasp is robust in the sense that the contacts would passively resist the given set of external wrenches without requiring active control, even under huge uncertainty in the contact parameters.

There are several natural paths for future research. First, our robust grasp synthesis approach is rather preliminary. For instance, we focused only on the selection of preload forces, while the contact locations should also be used as a design parameter. A more thorough approach requires formulation of coordinate invariant functions [16] that can measure the quality of a grasp arrangement based on its polyhedral bound. Using such functions, optimal grasps that robustly resist a collection of external wrenches can be systematically selected. Second, the quasi-rigid-body framework requires that the deformations be localized to the vicinity of the contacts. However, complex objects with slender substructures exhibit global deformations that can dominate the force-displacement relationship at the contacts. The modeling of such global deformations is a challenging problem which is currently under investigation. Third, we are in the process of clarifying the connection between the polyhedral bound and the notion of strong stability [30].

Finally, consider the extension of the polyhedral bound to three dimensions. If the fingers can only apply pure forces at point contacts, the basic relationships that led to the polyhedral bound in two dimensions also hold in three dimensions. For a $k$-contact grasp in three dimensions, $\Delta f$-space can be identified with a copy of $\mathbb{R}^{3 k}$ based at the preload forces $f_{0} \in \mathbb{R}^{3 k}$. The skeletal set $V_{0}$ is given by $V_{0}=\bar{G}^{T} \dot{q}$ such that $\dot{q} \in T_{q_{0}} \mathbb{R}^{6}$. The feasible set of force changes is given by $V=-\Sigma \bar{G}^{T} \dot{q}$ such that $\dot{q} \in T_{q_{0}} \mathbb{R}^{6}$ and $\Sigma$ contains the contact parameters. On the other hand, the contact forces induced by an external wrench $\boldsymbol{w}_{\text {ext }}$ satisfy the equilibrium equation $\bar{G}\left(\boldsymbol{f}_{0}+\Delta \boldsymbol{f}\right)+\boldsymbol{w}_{\text {ext }}=\overrightarrow{0}$, where $\boldsymbol{f}_{0}$ are the preload forces. Since the preload forces satisfy $\bar{G} f_{0}=\overrightarrow{0}$, the equilibrium condition defines an affine subspace $U$ in $\Delta f$-space. A key step is that here, too, $V_{0}$ is orthogonal to $U$ in $\Delta f$-space. If one makes the reasonable conjecture that $\Sigma$ is diagonal and nonnegative, the set $\mathcal{P}=U \cap V$ is again a bounded polyhedral set in $\Delta f$-space.

\section{APPENDIX}

\section{A. Details of the Micro-Penetration Principle}

This appendix contains a proof of the formula for the derivative of the normal penetration $\delta_{i}^{n}$. We need the following lemma.

Lemma A.1 ([33]): The overlap segment is perpendicular to the boundaries of $\mathcal{B}$ and $\mathcal{A}_{i}$ at its endpoints $x_{i}$ and $y_{i}$ (Fig. 2).

Lemma A.2: Let $\mathcal{A}_{i}$ be stationary and let $\mathcal{B}$ move along a c-space curve $q(t)$, such that $\mathcal{B}$ maintains a positive overlap with $\mathcal{A}_{i}$. Then $(d / d t) \delta_{i}^{n}(q(t))=-n_{i} \cdot \dot{X}_{r_{i}}(q(t))$, where $-n_{i}$ is the outward unit normal to $\mathcal{B}$ at $x_{i}(t)=X_{r_{i}}(q(t))$.

Proof: Let $x_{i}$ and $y_{i}$ be the endpoints of the overlap segment (Fig. 2), so that $\delta_{i}^{n}(q(t))=\left\|y_{i}(t)-x_{i}(t)\right\|$. Using Lemma A.l, the unit normal pointing into $\mathcal{B}$ is given by $n_{i}=\left(y_{i}-\right.$ $\left.x_{i}\right) /\left\|y_{i}-x_{i}\right\|$. Hence

$$
\frac{d}{d t} \delta_{i}^{n}(t)=\frac{1}{\left\|y_{i}-x_{i}\right\|}\left(y_{i}-x_{i}\right) \cdot\left(\dot{y}_{i}-\dot{x}_{i}\right)=n_{i} \cdot\left(\dot{y}_{i}-\dot{x}_{i}\right) .
$$

Since $\mathcal{A}_{i}$ is stationary and $y_{i}(t)$ lies on the boundary of $\mathcal{A}_{i}, \dot{y}_{i}$ is tangent to the boundary of $\mathcal{A}_{i}$ at $y_{i}$. However, $n_{i}$ is also normal 
to the boundary of $\mathcal{A}_{i}$ at $y_{i}$, according to Lemma A.1. Hence, $n_{i} \cdot \dot{y}_{i}=0$, and consequently, $(d / d t) \delta_{i}^{n}(q(t))=-n_{i} \cdot \dot{x}_{i}$. Now $x_{i}(q(t))=R(t) r_{i}(t)+d(t)$. Hence, $\dot{x}_{i}=\dot{X}_{r_{i}}+R \dot{r}_{i}$. Finally, it can be shown that the summand $R \dot{r}_{i}$ is tangent to the boundary of $\mathcal{B}$ at $x_{i}(t)$. Hence, $(d / d t) \delta_{i}^{n}(q(t))=-n_{i} \cdot \dot{X}_{r_{i}}$.

\section{B. Details of the Polyhedral-Bound Derivation}

This appendix contains several results which are used in the derivation of the polyhedral bound. The first lemma characterizes the feasible set $V$. Recall that the skeletal set of $V$ is the subset

$$
V_{0}=\left\{\left(\begin{array}{c}
\Delta f^{t} \\
\Delta f^{n}
\end{array}\right)=\bar{G}^{T} \dot{q}: \dot{q} \in T_{q_{0}} \mathbb{R}^{3}\right\}
$$

Recall, too, that a $p$-quadrant in $\mathbb{R}^{p}$ is the rectangular cone spanned by a particular choice of directions along the coordinate axes in this space.

Lemma B.1: Let the skeletal set $V_{0}$ be transversal to the coordinate hyperplanes in $\left(\Delta f^{t}, \Delta f^{n}\right)$-space (which is the generic case). Then, the feasible set $V$ consists of the interior of all $2 k$-quadrants in $\Delta f$-space which are intersected by $V_{0}$.

In the special case where $V_{0}$ is aligned with some coordinate hyperplane in $\left(\Delta f^{t}, \Delta f^{n}\right)$-space, $V$ fills the $2 k$-quadrants whose interior is intersected by $V_{0}$, as well as the $(2 k-1)$-quadrants of the coordinate hyperplane which is aligned with $V_{0}$.

Proof: Let $\dot{q} \in T_{q_{0}} \mathbb{R}^{3}$ be an instantaneous motion of $\mathcal{B}$ such that the vector $\bar{G}^{T} \dot{q} \in \mathbb{R}^{2 k}$ has nonzero components. Let $\bar{g}_{1}, \ldots, \bar{g}_{2 k} \in \mathbb{R}^{3}$ denote the rows of $\bar{G}^{T}$. Recall that the contact parameters are denoted collectively as $\sigma_{1}, \ldots, \sigma_{2 k}$. Since $\Sigma$ is diagonal with entries $\sigma_{1}, \ldots, \sigma_{2 k}$

$$
\Sigma \bar{G}^{T} \dot{q}=\sigma_{1}\left(\begin{array}{c}
\bar{g}_{1} \cdot \dot{q} \\
0 \\
\vdots \\
0
\end{array}\right)+\cdots+\sigma_{2 k}\left(\begin{array}{c}
0 \\
\vdots \\
0 \\
\bar{g}_{2 k} \cdot \dot{q}
\end{array}\right)
$$

where $\sigma_{1}, \ldots, \sigma_{2 k}>0$. It follows from (22) that the set $\Sigma \bar{G}^{T} \dot{q}$ is the interior of the $2 k$-quadrant spanned by the coordinate projections of $\bar{G}^{T} \dot{q}$ on the coordinate axes of $\left(\Delta f^{t}, \Delta f^{n}\right)$-space. In other words, the set $\Sigma \bar{G}^{T} \dot{q}$ fills the interior of the $2 k$-quadrant containing the vectors $\bar{G}^{T} \dot{q}$ such that $\dot{q} \in T_{q_{0}} \mathbb{R}^{3}$. This implies that $V$ fills the interior of the $2 k$-quadrants containing the skeletal set $V_{0}$.

Next, we provide a proof of Lemma 3.3 that $\mathcal{P}$ is always nonempty and connected.

Lemma 3.3: The polyhedral set $\mathcal{P}$ consists of a single connected component in $\Delta f$-space. Moreover, this set is always nonempty and contains the solution $-\bar{G}^{\dagger} \boldsymbol{w}_{\text {ext }}$.

Proof: Let $\mathcal{V} \subset \mathbb{R}^{2 k}$ be the set of positive parameters $\boldsymbol{\sigma}=$ $\left(\sigma_{1}, \ldots, \sigma_{2 k}\right)$ along the diagonal of $\Sigma$. First, we show that the collection of contact-force changes, $\left(\Delta f^{t}, \Delta f^{n}\right) \in \mathcal{P}$, is the image of $\mathcal{V}$ under a continuous mapping. Consider the equation representing the intersection of $U$ with $V$ as

$$
E \boldsymbol{\nu}-\bar{G}^{\dagger} \boldsymbol{w}_{\mathrm{ext}}=-\Sigma(\boldsymbol{\sigma}) \bar{G}^{T} \dot{q}
$$

where $\boldsymbol{\nu} \in \mathbb{R}^{m}, \dot{q} \in \mathbb{R}^{3}$, and $\boldsymbol{\sigma} \in \mathcal{V}$. Since $\Sigma(\boldsymbol{\sigma})$ is positive definite for $\sigma \in \mathcal{V}$, we can multiply both sides of (23) by $E^{T} \Sigma^{-1}(\boldsymbol{\sigma})$, to obtain an implicit description of $\mathcal{P}$ in $(\nu, \sigma)$-space

$$
E^{T} \Sigma^{-1}(\boldsymbol{\sigma})\left(E \boldsymbol{\nu}-\bar{G}^{\dagger} \boldsymbol{w}_{\text {ext }}\right)=\overrightarrow{0} .
$$

The matrix $E^{T} \Sigma^{-1}(\boldsymbol{\sigma}) E$ is positive definite for $\boldsymbol{\sigma} \in \mathcal{V}$. Hence, (24) can be solved for $\boldsymbol{\nu}$ as a function of $\boldsymbol{\sigma}$ as

$$
\boldsymbol{\nu}(\boldsymbol{\sigma})=\left[E^{T} \Sigma^{-1}(\boldsymbol{\sigma}) E\right]^{-1} E^{T} \Sigma^{-1}(\boldsymbol{\sigma}) \bar{G}^{\dagger} \boldsymbol{w}_{\mathrm{ext}} .
$$

The function $\nu(\sigma)$ is well-defined and continuous for $\sigma \in \mathcal{V}$. It follows that the function $E \boldsymbol{\nu}(\boldsymbol{\sigma})-\bar{G}^{\dagger} \boldsymbol{w}_{\text {ext }}$, which maps $\mathcal{V}$ to $\mathcal{P}$, is also well-defined and continuous. The image of a connected set under a continuous map is connected. Since $\mathcal{V}$ is nonempty and connected, the polyhedral set $\mathcal{P}$ is also nonempty and connected.

The following is a proof of Lemma 3.4, which is concerned with the planar facets of $\mathcal{P}$.

Lemma 3.4: The planar facets of $\mathcal{P}$ are embedded in the coordinate hyperplane of $\left(\Delta f^{t}, \Delta f^{n}\right)$-space. Moreover, using the null-space parametrization (10), the $i$ th facet of $\mathcal{P}$ satisfies the formula

$$
\boldsymbol{e}_{i} \cdot \boldsymbol{\nu}-\left(\bar{G}^{\dagger} \boldsymbol{w}_{\mathrm{ext}}\right)_{i}=0, \quad \text { where } \boldsymbol{\nu} \in \mathbb{R}^{m}
$$

where $e_{i} \in \mathbb{R}^{m}$ is the $i$ th row of $E$ and $\left(\bar{G}^{\dagger} \boldsymbol{w}_{\text {ext }}\right)_{i}$ is the $i$ th component of $\bar{G}^{\dagger} \boldsymbol{w}_{\text {ext }} \in \mathbb{R}^{2 k}$.

Proof: According to Lemma B.1, the feasible set $V$ consists of entire $2 k$-quadrants in $\Delta f$-space. The set $V$ is therefore bounded by the coordinate hyperplanes in $\Delta f$-space. On the other hand, $U$ is an affine subspace in $\Delta f$-space with no boundary points. Hence, all of the boundary points of $\mathcal{P}$ originate from the boundary of $V$, which is embedded in the coordinate hyperplanes of $\Delta f$-space.

Consider now (23), which represents the intersection of $U$ with $V$. By equating the $i$ th entry on the LHS of (23) to zero, we obtain a parametrization of the intersection of $U$ with the $i$ th coordinate hyperplanes in $\Delta f$-space. This is precisely formula (26) above.

Finally, we give a proof of Lemma 3.5, which characterizes the vertices and convex cells of $\mathcal{P}$.

Lemma 3.5: The set $\mathcal{P}$ is a union of convex polyhedra in $\Delta f$-space. The number of convex polyhedra is bounded from above by $(2 k)^{2}$, and the total number of vertices is bounded from above by $(2 k)^{3}$, where $k$ is the number of contacts.

According to Lemma B.l, the feasible set $V$ fills entire $2 k$-quadrants in $\Delta f$-space. Let $K_{i}$ denote a $2 k$-quadrant and let $\mathcal{I}$ denote the index set of the $2 k$-quadrants occupied by $V$. The set $\mathcal{P}$ can be written as the union $\mathcal{P}=U \cap V=\cup_{i \in \mathcal{I}} U \cap K_{i}$. Each intersection $U \cap K_{i}$ is a convex polyhedron in $\Delta f$-space, since both $U$ and $K_{i}$ are convex polyhedra. Hence $\mathcal{P}$ is a union of convex polyhedra in $\Delta f$-space.

Next, consider the number of convex polyhedra in $\mathcal{P}$. Lemma $B .1$ asserts that the feasible set fills the $2 k$-quadrants which are intersected by the skeletal set $V_{0}$. Hence, the number of convex polyhedra in $\mathcal{P}$ is bounded from above by the number of $2 k$-quadrants intersected by $V_{0}$. The set $V_{0}$ is given by

$$
V_{0}=\left\{\left(\begin{array}{c}
\Delta f^{t} \\
\Delta f^{n}
\end{array}\right)=\bar{G}^{T} \dot{q}: \dot{q} \in T_{q_{0}} \mathbb{R}^{3}\right\}
$$


where $T_{q_{0}} \mathbb{R}^{3}$ can be identified with $\mathbb{R}^{3}$. When $V_{0}$ crosses a coordinate hyperplane in $\Delta f$-space, some entry in $\bar{G}^{T} \dot{q}$ vanishes. Letting $\bar{g}_{i}$ denote the $i$ th row of $\bar{G}^{T}$, the vanishing of the $i$ th entry is given by $\bar{g}_{i} \cdot \dot{q}=0$. This equation defines a plane through the origin in $\mathbb{R}^{3}$. Since $\bar{G}^{T}$ is $2 k \times 3$, there are $2 k$ such planes in $\mathbb{R}^{3}$. The planes partition $\mathbb{R}^{3}$ into polyhedral cones having a common vertex at the origin. Each polyhedral cone corresponds to a particular $2 k$-quadrant occupied by $V_{0}$ in $\Delta f$-space. The number of polyhedral cones can be bounded by considering the unit sphere in $\mathbb{R}^{3}$, denoted $S^{2}$. The intersection of two planes, being an infinite line through the origin, induces two antipodal points on $S^{2}$. Hence the total number of vertices induced on $S^{2}$ by the planes is twice the number of ways to select two out of $2 k$ planes. The number of vertices in $S^{2}$ is therefore bounded by $2((2 k) !) /(2 !(2 k-2) !)=2 k(2 k-1)$. Using the fact that every vertex is generated by the intersection of two circles in $S^{2}$, an argument based on the Euler characteristic of $S^{2}$ shows that the total number of spherical cells induced by the $2 k$ planes is bounded by $2+2 k(2 k-1) \leq(2 k)^{2}$. This number is an upper bound on the number of $2 k$-quadrants intersected by $V_{0}$ in $\Delta f$-space.

Last consider the number of vertices in $\mathcal{P}$. According to Lemma 3.4, each planar facet of $\mathcal{P}$ is embedded in a particular coordinate hyperplane of $\left(\Delta f^{t}, \Delta f^{n}\right)$-space. Since $\mathcal{P}$ is generically $m$-dimensional, each vertex of $\mathcal{P}$ lies at the intersection of $m$ coordinate hyperplanes in $\Delta f$-space with the affine subspace $U$. Moreover, each $m$-touple of coordinate hyperplanes can intersect the affine subspace $U$ at most at one point. Thus, the total number of vertices in $\mathcal{P}$ is bounded from above by the number of $m$-touples of coordinate hyperplanes in $\mathbb{R}^{2 k}$. Since $m=2 k-3$, the number of $m$-tuples of coordinate hyperplanes is $((2 k) !) /((2 k-m) ! m !)=((2 k) !) /(3 !(2 k-3) !)=$ $(1 / 6) 2 k(2 k-1)(2 k-2) \leq(2 k)^{3}$.

\section{Computation of the Polyhedral Bound}

This Appendix sketches an algorithm that computes the set $\mathcal{P}$ as a union of convex polyhedra. The algorithm is based on the proof of Lemma 3.5, which identifies the $2 k$-quadrants occupied by $V$ with spherical cells on the unit sphere. A key ingredient in this algorithm is a procedure that checks if an affine subspace $W \subset \mathbb{R}^{p}$ intersects a given $p$-quadrant in $\mathbb{R}^{p}$. First we define some terminology. Let $K_{i}$ denote a $p$-quadrant in $\mathbb{R}^{p}$. Since $K_{i}$ is spanned by a particular choice of coordinate directions in $\mathbb{R}^{p}$, it is uniquely identified by a sign vector $Z_{i}=( \pm 1, \ldots, \pm 1)$ according to the coordinate-axes directions in $K_{i}$. Let $\vec{x}=$ $\left(x_{1}, \ldots, x_{p}\right)$ denote the coordinates of $\mathbb{R}^{p}$. Then checking that $W$ intersects a $p$-quadrant $K_{i}$ amounts to checking that the following set of linear inequalities is nonempty:

$$
\left(\begin{array}{c}
Z_{i 1} x_{1} \\
\vdots \\
Z_{i p} x_{p}
\end{array}\right) \geq\left(\begin{array}{c}
0 \\
\vdots \\
0
\end{array}\right) \text {, such that } x=\left(\begin{array}{c}
x_{1} \\
\vdots \\
x_{p}
\end{array}\right) \in W
$$

where $Z_{i}=\left(Z_{i 1}, \ldots, Z_{i p}\right)$ is the sign vector of $K_{i}$. The problem (27) is a linear-programming problem that can be solved in $O(p)$ steps using interior point methods [5].
From now on we assume the availability of a procedure $\operatorname{ASK}(p, W, Z)$, that returns a yes-answer if the affine subspace $W$ intersects the $p$-quadrant with a sign vector $Z$.

The algorithm runs in $\Delta f$-space which is identified here with $\mathbb{R}^{2 k}$, where $k$ is the number of contacts. Assuming that the $2 k$-quadrants of $\Delta f$-space are indexed by some method, the algorithm maintains the following data structures. The set $\mathcal{J}$ contains the indices of the $2 k$-quadrants occupied by the feasible set $V$. The set $\mathcal{I} \subset \mathcal{J}$ contains the indices of the $2 k$-quadrants which are additionally intersected by the affine subspace $U$. The algorithm stores its output in lists $L_{i}$ such that $i \in \mathcal{I}$. These lists contain the linear inequalities that bound the convex pieces of $\mathcal{P}$.

Polyhedral Bound Algorithm:

Input: Grasp matrix $\bar{G}$ with columns $\bar{g}_{1}, \ldots, \bar{g}_{2 k} \in \mathbb{R}^{3}$, an external wrench $\boldsymbol{w}_{\text {ext }}$.

$\underline{\text { Data }}$ Structures: Initially empty index sets $\mathcal{I}$ and $\mathcal{J}$, initially empty lists $L_{i}$.

1) Construct the null-space matrix $E$ and the affine subspace $U$ in $\mathbb{R}^{2 k}$.

2) For each $\left(\bar{g}_{i_{1}}, \bar{g}_{i_{2}}\right)$, construct two vertices on the unit sphere $u_{i_{1} i_{2}}= \pm\left(\bar{g}_{i_{1}} \times \bar{g}_{i_{2}}\right) /\left\|\bar{g}_{i_{1}} \times \bar{g}_{i_{2}}\right\|$.

3) Connect each vertex $u_{i_{1} i_{2}}$ with the circular edges induced by the planes $\bar{g}_{i_{1}} \cdot \dot{q}=0, \bar{g}_{i_{2}} \cdot \dot{q}=0$.

4) Using a line-sweep algorithm, compute a sample point $s_{i} \in \mathbb{R}^{3}$ in each spherical cell.

5) For each spherical cell, determine the sign vector $Z_{i}$ of the $2 k$-quadrant associated with the cell, according to the sign of $\bar{G}^{T} s_{i}$. Add to $\mathcal{J}$ the index of the $2 k$-quadrant.

6) For each $2 k$-quadrant $K_{i}$ in $\mathcal{J}$ :

6.1 call $\operatorname{ASK}\left(2 k, U, Z_{i}\right)$ to check if $K_{i}$ is intersected by affine subspace $U$.

6.2 If it does, add $i$ to the index-set $\mathcal{I}$.

7) For each $2 k$-quadrant $K_{i}$ in $\mathcal{I}$ :

7.1 Let $U_{j}$ be the intersection of $U$ with the $j$ th coordinatehyperplane in $\mathbb{R}^{2 k}$.

7.2 For each (2k-1)-quadrant $K_{i j}$ on the boundary of $K_{i}$ :

7.2.1 Call $\operatorname{ASK}\left(2 k-1, U_{j}, Z_{i j}\right)$ to check if $K_{i j}$ is intersected by $U_{j}$.

7.2.2 If it does, add the index $j$ to the list $L_{i}$.

Output: For each $i \in \mathcal{I}$, a list $L_{i}$ of coordinate-hyperplanes containing the facets of $K_{i} \cap \mathcal{P}$.

Each list $L_{i}$ determines one convex piece of $\mathcal{P}$. Using the null-space parametrization (10), each of these convex pieces is given by

$K_{i} \cap \mathcal{P}=\cap_{j \in L_{i}}\left(Z_{i j} \cdot\left(\boldsymbol{e}_{j} \cdot \boldsymbol{\nu}-\left(\bar{G}^{\dagger} \boldsymbol{w}_{\mathrm{ext}}\right)_{j}\right)>0\right)$ where $\boldsymbol{\nu} \in \mathbb{R}^{m}$.

In this formula, $Z_{i j}$ is the $j$ th component of the sign-vector $Z_{i}$ of $K_{i}, \boldsymbol{e}_{j} \in \mathbb{R}^{m}$ is the $j$ th row of $E$, and $\left(\bar{G}^{\dagger} \boldsymbol{w}_{\text {ext }}\right)_{j}$ is the $j$ th component of $\bar{G}^{\dagger} \boldsymbol{w}_{\text {ext }} \in \mathbb{R}^{2 k}$. Finally, the set $\mathcal{P}$ is the union $\mathcal{P}=\cup_{i \in \mathcal{I}} K_{i} \cap \mathcal{P}$.

The computational complexity of the algorithm is as follows. In step 4, the arrangement of cells on the unit sphere contains $O\left(k^{2}\right)$ cells (Lemma 3.5). Hence, a line sweep algorithm would identify a sample point per cell in $O\left(\log (k) k^{2}\right)$ time. At this stage, the index set $\mathcal{J}$ contains $O\left(k^{2}\right)$ indices. Step 6 invokes a linear program for each $2 k$-quadrant in $\mathcal{J}$, and this step takes $O\left(k^{3}\right)$ time to complete. In worst case, the reduced index set $\mathcal{I}$ 
still contains $O\left(k^{2}\right)$ indices. At the last stage, the algorithm determines which coordinate hyperplanes bound the intersection $K_{i} \cap U$ for each $K_{i}$ such that $i \in \mathcal{I}$. This inquiry involves execution of a linear program $2 k$ times per $K_{i}$. Since there are $O\left(k^{2}\right)$ indices in $\mathcal{I}$, this last stage takes $O\left(k^{4}\right)$ time. The computational complexity of the algorithm is therefore $O\left(k^{4}\right)$.

\section{REFERENCES}

[1] A. Ben-Isreal, "Notes on linear inequalities. I: The intersection of the nonnegative orthant with complementary orthogonal subspaces," $J$. Math. Anal. Appl., vol. 9, pp. 303-314, 1964.

[2] A. Bicchi, "On the problem of decomposing grasp and manipulation forces in multiple whole-limb manipulation," Int. J. Robot. Auton. Syst., vol. 13, no. 4, pp. 127-147, 1994.

[3] — , "On the closure properties of robotic grasping," Int. J. Robot. Res., vol. 14 , no. 4, pp. 319-334, 1995.

[4] _ - "Hands for dexterous manipulation and robust grasping: A difficult road toward simplicity," IEEE Trans. Robot. Autom., vol. 16, no. 6, pp. 652-662, Dec. 2000.

[5] S. P. Boyd and C. H. Barratt, Linear Controller Design. Englewood Cliffs, NJ: Prentice-Hall, 1991

[6] M. Buss, L. Faybusovich, and J. Moore, "Dikin-type algorithms for dextrous grasping force optimization," Int. J. Robot. Res., vol. 17, no. 8, pp. 311-334, 1998.

[7] M. R. Cutkosky and I. Kao, "Computing and controlling the compliance of a robotic hand," IEEE Trans. Robot. Autom., vol. 5, no. 2, pp. 151-165, Apr. 1989.

[8] D. Ding, Y. Liu, and Y. Wang, "Automatic selection of fixturing surfaces and fixturing points for polyhedral workpieces," IEEE Trans. Robot. Autom., vol. 17, no. 6, pp. 833-841, Dec. 2001.

[9] P. E. Dupont, "The effect of Coulomb friction on the existence and uniqueness of the forward dynamics problem," in Proc. IEEE Int. Conf. Robot. Autom., 1992, pp. 1442-1447.

[10] D. Elata and J. G. Berryman, "Contact force-displacement laws and the mechanical behavior of random packs of identical spheres," Mech. Mater, vol. 24, pp. 229-240, 1996

[11] F. Fen, M. Shoham, and R. Longman, "Lyapunov stability of force-controlled grasps with a multifingered hand," Int. J. Robot. Res., vol. 15, no. 2, pp. 137-154, 1996.

[12] H. Hertz, "On the contact of elastic solids," in Miscellaneous Papers by H. Hertz (1882). London, U.K.: Macmillan, 1896.

[13] W. S. Howard and V. Kumar, "On the stability of grasped objects," IEEE Trans. Robot. Autom., vol. 12, no. 6, pp. 904-917, Dec. 1996.

[14] Z. Ji and B. Roth, "Direct computation of grasping force for three-finger tip-prehension grasps," J. Mech., Transmiss., Autom. Des., vol. 110, pp. 405-413, 1988.

[15] K. L. Johnson, Contact Mechanics. Cambridge, U.K.: Cambridge Univ. Press, 1985.

[16] Q. Lin and J. W. Burdick, "Objective and frame-invariant kinematic metric functions for rigid bodies," Int. J. Robot. Res., vol. 19, no. 6, pp. 612-626, 2000.

[17] G. F. Liu, J. Li, and Z. X. Li, "Coordinated manipulation of objects by multifingered robotic hand in contact space and active joint space," in Proc. IEEE Int. Conf. Robot. Autom., 2002, pp. 3743-3748.

[18] Y.-H. Liu, "Qualitative test and force optimization of 3-D frictional form-closure grasps using linear programming," IEEE Trans. Robot. Autom., vol. 15, no. 1, pp. 163-173, Feb. 1999.

[19] P. Lotstedt, "Coulomb friction in two-dimensional rigid body systems," Zeitschrift fur Angewandte Mathematik und Mechanik, vol. 61, pp. 605-615, 1981.

[20] X. Markenscoff, L. Ni, and C. H. Papadimitriou, "The geometry of grasping," Int. J. Robot. Res., vol. 9, no. 1, pp. 61-74, 1990.

[21] X. Markenscoff and C. H. Papadimitriou, "Optimum grip of a polygon," Int. J. Robot. Res., vol. 8, no. 2, pp. 17-29, 1989.

[22] R. D. Mindlin and H. Deresiewicz, "Elastic spheres in contact under varying oblique forces," ASME J. Appl. Mech., vol. 20, pp. 327-344, 1953.

[23] B. Mirtich and J. F. Canny, "Easily computable optimum grasps in 2-D and 3-D," in Proc. IEEE Int. Conf. Robot. Autom., 1994, pp. 739-747.

[24] K. Mirza and D. E. Orin, "General formulation for force distribution in power grasps," in Proc. IEEE Int. Conf. Robot. Autom., 1994, pp. 880-887.

[25] B. Mishra, "Grasp metrics: Optimality and complexity," in Proc. Workshop Algorithmic Found. Robot. , 1995, pp. 137-165.
[26] R. M. Murray, Z. Li, and S. S. Sastry, A Mathematical Introduction to Robotic Manipulation. Boca Raton, FL: CRC, 1994.

[27] V.-D. Nguyen, "Constructing stable grasps," Int. J. Robot. Res., vol. 8, no. 1 , pp. 27-37, 1988.

[28] T. Omata, "Rigid body analysis of power grasps: Bounds of the indeterminate grasp force," in Proc. IEEE Int. Conf. Robot. Autom., 2001, pp 2203-2209.

[29] T. Omata and K. Nagata, "Rigid body analysis of the indeterminate grasp force in power grasps," IEEE Trans. Robot. Autom., vol. 16, no. 1, pp. 46-54, Feb. 2000.

[30] J. S. Pang and J. C. Trinkle, "Stability characterizations of rigid body contact problems with coulomb friction," Zeitschrift fur Angewandte Mathematik und Mechanik, vol. 80, no. 10, pp. 643-663, 2000.

[31] J. Park, K. Harada, and M. Kaneko, "Enveloping grasp feasibility inequality," in Proc. IEEE Int. Conf. Robot. Autom., 2001, pp. 2210-2216.

[32] T. Patterson and H. Lipkin, "Structure of robot compliance," ASME J. Mech. Des., vol. 115, no. 3, pp. 576-580, 1993.

[33] E. Rimon and J. W. Burdick, "Mobility of bodies in contact-II: How forces are generated by curvature effects," IEEE Trans. Robot. Autom., vol. 14, no. 5, pp. 709-717, Oct. 1998.

[34] E. Rimon, J. W. Burdick, and T. Omata. (2003, Jul.) A polyhedral bound on the indeterminate contact forces in planar fixturing and grasping arrangements. Tech. Rep., Dept. Mech. Eng., California Inst. Technol., Pasadena. [Online]. Available: http://robotics.caltech.edu/papers

[35] S. P. Timoshenko and J. N. Goodier, Theory of Elasticity, 3rd ed. New York: McGraw-Hill, 1969.

[36] J. C. Trinkle, "A quantitative test for form closure grasps," in Proc. IEEE/RSJ Int. Conf. Intell. Robots Syst., 1992, pp. 1670-1677.

[37] K. Walton, "The oblique compression of two elastic spheres," J. Mech Phys. Solids, vol. 26, pp. 139-150, 1978.

[38] J. D. Wolter and J. C. Trinkle, "Automatic selection of fixture points for frictionless assemblies," in Proc. IEEE Int. Conf. Robot. Autom., 1994 pp. 528-534.

[39] C.-H. Xiong, Y.-F. Li, H. Ding, and Y.-L. Xiong, "On the dynamic stability of grasping," Int. J. Robot. Res., vol. 18, no. 9, pp. 951-958, 1999.

[40] J. J. Xu, G. F. Liu, and Z. X. Li, "On quality functions for grasp synthesi and fixture planning," in Proc. IEEE Int. Conf. Robot. Autom., 2004, pp. 333-338.

[41] N. Xydas and I. Kao, "Modeling of contact mechanics and friction-limitsurfaces for soft fingers in robotics, with experimental results," Int. J. Robot. Res., vol. 18, no. 8, pp. 941-950, 1999.

[42] N. Xydas and I. Kao, "Study of soft-finger contact mechanics using finite elements analysis and experiments," Tech. Rep., Manuf. Autom. Lab., Dept. Mech. Eng., SUNY, Stony Brook, NY, 2000.

[43] T. Yoshikawa, "Passive and active closure by constraining mechanisms," J. Dyn. Syst., Meas., Control, vol. 121, no. 3-4, pp. 418-424, 1999.

Elon Rimon (M'96) received the B.Sc. degree in computer engineering from the Technion, Israel Institute of Technology, Haifa, Israel, in 1985, and the Ph.D. degree in electrical engineering from Yale University, New Haven, CT, in 1990

He has been with the Department of Mechanical Engineering, the Technion, since 1994, where he is currently an Associate Professor. His research interests include computational geometry algorithms in robotics, sensor-based motion planning, mobile robot problems, grasping and fixturing, and robotic locomotion.

Joel W. Burdick received the B.S. degree in mechanical engineering and chemistry from Duke University, Chapel Hill, NC, and the M.S. and Ph.D. degrees in mechanical engineering from Stanford University, Stanford, CA.

He joined the Department of Mechanical Engineering, California Institute of Technology, Pasadena, in May 1988, where he is currently a Professor of mechanical engineering and bioengineering. His current interests include robotic locomotion, sensor-based robot motion planning, multifingered robotic grasping, neural prosthetics, and applied nonlinear control theory.

Toru Omata (M'95) received the B.S., M.S., and Ph.D. degrees in control engineering from the Tokyo Institute of Technology, Tokyo, Japan, in 1981, 1983, and 1986, respectively.

From 1986 to 1994, he was a Researcher with the Electrotechnical Laboratory, Tsukuba, Japan. Since 1994, he has been an Associate Professor with the Tokyo Institute of Technology. His research interests include dexterous manipulation, multifingered hands, parallel robots, and legged robots. 